\title{
International consensus on severe lung cancer-the first edition
}

\author{
Chengzhi Zhou ${ }^{1 \#}$, Shiyue $\mathrm{Li}^{1 \#}$, Jun Liu ${ }^{1 \#}$, Qian $\mathrm{Chu}^{2 *}$, Liyun Miao ${ }^{3 \#}$, Linbo Cai ${ }^{4}$, Xiuyu Cai ${ }^{5}$, Yu Chen ${ }^{1}$,
} Fei Cui ${ }^{1}$, Yuchao Dong ${ }^{6}$, Wen Dong ${ }^{7}$, Wenfeng Fang ${ }^{8}$, Yong $\mathrm{He}^{9}$, Weifeng $\mathrm{Li}^{10}$, Min $\mathrm{Li}^{11}$, Wenhua Liang ${ }^{1}$, Gen Lin ${ }^{12}$, Jie Lin ${ }^{13}$, Xinqing Lin ${ }^{1}$, Hongbing Liu ${ }^{14}$, Ming Liu ${ }^{1}$, Xinlin Mu${ }^{15}$, Yi Hu ${ }^{16}$, Jie $\mathrm{Hu}^{17}$, Yang Jin ${ }^{18}$, Ziming Li ${ }^{19}$, Yinyin Qin ${ }^{1}$, Shengxiang Ren ${ }^{20}$, Gengyun Sun ${ }^{21}$, Yihong Shen ${ }^{22}$, Chunxia Su ${ }^{23}$, Kejing Tang ${ }^{24}$, Lin $\mathrm{Wu}^{25}$, Mengzhao Wang ${ }^{26}$, Huijuan Wang ${ }^{27}$, Kai Wang ${ }^{28}$, Yuehong Wang ${ }^{22}$, Ping Wang ${ }^{29}$, Hongmei Wang $^{30}$, Qi Wang ${ }^{31}$, Zhijie Wang ${ }^{32}$, Xiaohong Xie ${ }^{1}$, Zhanhong Xie ${ }^{1}$, Xin Xu ${ }^{1}$, Fei Xu ${ }^{33}$, Meng Yang ${ }^{34}$, Boyan Yang ${ }^{35,36}$, Xiangjun $\mathbf{Y i}^{37}$, Xiaoqun Ye ${ }^{38}$, Feng Ye $^{39}$, Zongyang Yu ${ }^{40}$, Dongsheng Yue ${ }^{41}$, Bicheng Zhang ${ }^{42}$, Jian Zhang ${ }^{43}$, Jianqing Zhang ${ }^{44}$, Xiaoju Zhang ${ }^{45}$, Wei Zhang ${ }^{46}$, Wei Zhao ${ }^{47}$, Bo Zhu ${ }^{48}$, Zhengfei Zhu ${ }^{49}$, Wenzhao Zhong ${ }^{50}$, Chunxue Bai ${ }^{51}$, Liangan Chen ${ }^{52}$, Baohui Han ${ }^{53}$, Chengping $\mathrm{Hu}^{54}$, Shun $\mathrm{Lu}^{55}$, Weimin $\mathrm{Li}^{56}$, Yong Song ${ }^{57}$, Jie Wang ${ }^{58}$, Caicun Zhou ${ }^{59}$, Jianying Zhou ${ }^{60}$, Yanbin Zhou ${ }^{61}$, Yuichi Saito $^{62}$, Yoshinobu Ichiki ${ }^{63}$, Hitoshi Igai ${ }^{64}$, Satoshi Watanabe ${ }^{65}$, Sara Bravaccini ${ }^{66}$, Alfonso Fiorelli ${ }^{67}$, Francesco Petrella ${ }^{68,69}$, Takeo Nakada ${ }^{70}$, Piergiorgio Solli ${ }^{71}$, Nikolaos Tsoukalas ${ }^{72}$, Yuki Kataoka ${ }^{73}$, Taichiro Goto $^{74}$, Rossana Berardi ${ }^{75}$, Jianxing $\mathbf{H e}^{1}$, Nanshan Zhong ${ }^{1}$

${ }^{1}$ State Key Laboratory of Respiratory Disease, National Clinical Research Centre for Respiratory Disease, Guangzhou Institute of Respiratory Health, The First Affiliated Hospital, Guangzhou Medical University, Guangzhou, China; ${ }^{2}$ Department of Oncology, Tongji Hospital, Huazhong University of Science and Technology, Wuhan, China; ${ }^{3}$ Department of Respiratory and Critical Care Medicine, Nanjing Drum Tower Hospital, The Affiliated Hospital of Nanjing University Medical School, Nanjing, China; ${ }^{4}$ Department of Oncology, Guangdong Sanjiu Brain Hospital, Guangzhou, China; ${ }^{5}$ Department of General Internal Medicine, State Key Laboratory of Oncology in South China, Sun Yat-sen University Cancer Center, Guangzhou, China; ${ }^{6}$ Department of Pulmonary and Critical Care Medicine, Shanghai Changhai Hospital, Second Military Medical University, Shanghai, China; ${ }^{7}$ Department of Oncology, Hainan Cancer Hospital, Haikou, China; ${ }^{8}$ Department of Medical Oncology, State Key Laboratory of Oncology in South China, Collaborative Innovation Center for Cancer Medicine, Sun Yat-sen University Cancer Center, Guangzhou, China; ${ }^{9}$ Department of Respiratory Medicine, Daping Hospital, Army Medical University, Chongqing, China; ${ }^{10}$ Department of Respiratory Medicine, General Hospital of Guangzhou Military Command of PLA, Guangzhou, China; ${ }^{11}$ Department of Respiratory Medicine, Xiangya Cancer Center, Xiangya Hospital, Central South University, Changsha, China; ${ }^{12}$ Department of Thoracic Oncology, Fujian Cancer Hospital and Fujian Medical University Cancer Hospital, Fuzhou, China; ${ }^{13}$ Department of Medical Oncology, the Second Affiliated Hospital of Kunming Medical University, Kunming, China; ${ }^{14}$ Department of Respiratory Medicine, Jinling Hospital, Nanjing University School of Medicine, Nanjing, China; ${ }^{15}$ Department of Respiratory and Critical Care Medicine, Peking University People's Hospital, Beijing, China; ${ }^{16}$ Department of Medical Oncology, Chinese People's Liberation Army (PLA) General Hospital, Beijing, China; ${ }^{17}$ Department of Respiratory Medicine, Zhongshan Hospital, Fudan University, Shanghai, China; ${ }^{18}$ Department of Respiratory and Critical Care Medicine, NHC Key Laboratory of Pulmonary Diseases, Union Hospital, Tongji Medical College, Huazhong University of Science and Technology, Wuhan, China; ${ }^{19}$ Shanghai Lung Cancer Center, Shanghai Chest Hospital, Shanghai Jiao Tong University, Shanghai, China; ${ }^{20}$ Department of Medical Oncology, Shanghai Pulmonary Hospital, Thoracic Cancer Institute, Tongji University School of Medicine, Shanghai, China; ${ }^{21}$ Department of Respiratory and Critical Care Medicine, The First Affiliated Hospital of Anhui Medical University, Hefei, China; ${ }^{22}$ Department of Respiratory Disease, The First Affiliated Hospital, College of Medicine, Zhejiang University, Hangzhou, China; ${ }^{23}$ Department of Medical Oncology, Shanghai Pulmonary Hospital, Tongji University School of Medicine, Shanghai, China; ${ }^{24}$ Division of Pulmonary and Critical Care Medicine, The First Affiliated Hospital of Sun Yat-sen University, Institute of Pulmonary Diseases, Sun Yat-sen University, Guangzhou, China; ${ }^{25}$ Thoracic Medicine Department II, Hunan Cancer Hospital, Changsha, China; ${ }^{26}$ Department of Respiratory and Critical Care Medicine, Peking Union Medical College Hospital, Beijing, China; ${ }^{27}$ Department of Medical Oncology, The Affiliated Cancer Hospital of Zhengzhou University, Henan Cancer Hospital, Zhengzhou, China; ${ }^{28}$ Department of Respiratory Medicine, Fourth Affiliated Hospital Zhejiang University School of Medicine, Hangzhou, China; ${ }^{29}$ Department of Respiratory and Critical Care Medicine, the Eighth Medical Center of PLA General Hospital, Beijing, China; ${ }^{30}$ Department of Respiratory and Critical Care Medicine, The Affiliated Hospital of Qingdao University, Qingdao, China; ${ }^{31}$ Department of Respiratory Medicine, The Second Affiliated Hospital of Dalian Medical University, Dalian, China; ${ }^{32}$ National Cancer Center/National Clinical Research Center for Cancer/Cancer Hospital, Chinese Academy of Medical Sciences and Peking Union Medical College, Beijing, China; ${ }^{33}$ Department of Respiratory Medicine, The First Affiliated Hospital of Nanchang University, Nanchang, China; ${ }^{34}$ Department of Respiratory Disease, China-Japan Friendship Hospital, Beijing, China; ${ }^{35}$ Department of 
Comprehensive Oncology, National Cancer Center/National Clinical Research Center for Cancer/Cancer Hospital \& Shenzhen Hospital, Chinese Academy of Medical Sciences and Peking Union Medical College, Shenzhen, China; ${ }^{36}$ Department of Comprehensive Oncology, National Cancer Center/National Clinical Research Center for Cancer/Cancer Hospital, Chinese Academy of Medical Sciences and Peking Union Medical College, Beijing, China; ${ }^{37}$ Department of Medical Oncology, The First Affiliated Hospital of Nanchang University, Nanchang, China; ${ }^{38}$ Department of Respiratory Diseases, The Second Affiliated Hospital of Nanchang University, Nanchang, China; ${ }^{39}$ Department of Medical Oncology, The first affiliated hospital of Xiamen University, Xiamen, China; ${ }^{40}$ Department of Pulmonary and Critical Care Medicine, The ${ }^{900}$ th Hospital of Joint Logistic Support Force, PLA, Fuzhou, China; ${ }^{41}$ Department of Lung Cancer, Tianjin Lung Cancer Center, Tianjin Medical University Cancer Institute and Hospital, National Clinical Research Center for Cancer, Key Laboratory of Cancer Prevention and Therapy, Tianjin's Clinical Research Center for Cancer, Tianjin, China; ${ }^{42}$ Cancer Center, Renmin Hospital of Wuhan University, Wuhan, China; ${ }^{43}$ Department of Pulmonary and Critical Care Medicine, Xijing Hospital, Fourth Military Medical University, Xi'an, China; ${ }^{44}$ Second Department of Respiratory and Critical Care Medicine, The First Affiliated Hospital of Kunming Medical University, Kunming, China; ${ }^{45}$ Department of Respiratory and Critical Care Medicine, Henan Provincial People's Hospital, People's Hospital of Zhengzhou University, Zhengzhou, China; ${ }^{46}$ Department of Respiratory and Critical Care Medicine, The First Affiliated Hospital of Nanchang University, Nanchang, China; ${ }^{47}$ Department of Pulmonary and Critical Care Medicine, The General Hospital of People's Liberation Army, Beijing, China; ${ }^{48}$ Institute of Cancer, Xinqiao Hospital, Army Medical University, Chongqing, China; ${ }^{49}$ Department of Radiation Oncology, Fudan University Shanghai Cancer Center, Shanghai, China; ${ }^{50}$ Guangdong Lung Cancer Institute, Guangdong General Hospital, and Guangdong Academy of Medical Sciences, Guangzhou, China; ${ }^{51}$ Department of Pulmonary Medicine, Zhongshan Hospital, Fudan University, Shanghai, China; ${ }^{52}$ Department of Respiratory, Chinese People's Liberation Army (PLA) General Hospital, Beijing, China; ${ }^{53}$ Department of Pulmonology, Shanghai Chest Hospital, Shanghai, China; ${ }^{54}$ Department of Pulmonary Medicine, Xiangya Hospital, Central South University, Changsha, China; ${ }^{55}$ Department of Oncology, Shanghai Lung Cancer Center, Shanghai Chest Hospital, Shanghai Jiao Tong University, Shanghai, China; ${ }^{56}$ Department of Respiratory and Critical Care Medicine, Clinical Research Center for Respiratory Disease, West China Hospital, Sichuan University, Chengdu, China; ${ }^{57}$ Department of Respiratory and Critical Care Medicine, Jinling Hospital, Nanjing, China; ${ }^{58}$ Key Laboratory of Respiratory Disease Pathogenomics, Chinese Academy of Medical Sciences and Peking Union Medical College, Beijing, China; ${ }^{59}$ Department of Oncology, Shanghai Pulmonary Hospital, Tongji University School of Medicine, Shanghai, China; ${ }^{60}$ Department of Respiratory Diseases, The First Affiliated Hospital of College of Medicine, Zhejiang University, Hangzhou, China; ${ }^{61}$ Department of Internal Medicine, The First Affiliated Hospital, Sun Yat-sen University, Guangzhou, China; ${ }^{62}$ Department of Surgery, Teikyo University School of Medicine, Tokyo, Japan; ${ }^{63}$ Department of General Thoracic Surgery, National Hospital Organization, Saitama Hospital, Wako, Japan; ${ }^{64}$ Department of General Thoracic Surgery, Japanese Red Cross Maebashi Hospital, Maebashi, Gunma, Japan; ${ }^{65}$ Department of Respiratory Medicine and Infectious Diseases, Niigata University Graduate School of Medical and Dental Sciences, Niigata, Japan; ${ }^{66}$ IRCCS Istituto Romagnolo per lo Studio dei Tumori (IRST) "Dino Amadori”, Meldola, Italy; ${ }^{67}$ Thoracic Surgery Unit, Universitàdella Campania Luigi Vanvitelli, Naples, Italy; ${ }^{68}$ Division of Thoracic Surgery, IRCCS European Institute of Oncology, Milan, Italy; ${ }^{69}$ Department of Oncology and Hemato-oncology, University of Milan, Milan, Italy; ${ }^{70}$ Division of Thoracic Surgery, Department of Surgery, the Jikei University School of Medicine, Tokyo, Japan; ${ }^{71}$ Department of Cardio-Thoracic Surgery and Hearth \& Lung Transplantation, IRCCS Azienda Ospedaliero-Universitaria di Bologna, Bologna, Italy; ${ }^{72}$ Department of Oncology, 401 General Military Hospital, Athens, Greece; ${ }^{73}$ Department of Internal Medicine, Kyoto Min-Iren Asukai Hospital, Kyoto, Japan; ${ }^{74}$ Lung Cancer and Respiratory Disease Center, Yamanashi Central Hospital, Yamanashi, Japan; ${ }^{75}$ Clinica Oncologica, Università Politecnica delle Marche, Azienda Ospedaliero-Universitaria Ospedali Riuniti Umberto I, GM Lancisi, G Salesi di Ancona, Italy

\#These authors contributed equally to this work.

Correspondence to: Jianxing He; Nanshan Zhong. Guangzhou Institute of Respiratory Health, State Key Laboratory of Respiratory Disease, First Affiliated Hospital, Guangzhou Medical University, 151 Yanjiang Road, Guangzhou 510120, China. Email: drjianxing.he@gmail.com; nanshan@vip.163.com.

Submitted Mar 10, 2021. Accepted for publication Jun 17, 2021.

doi: $10.21037 /$ tlcr-21-467

View this article at: https://dx.doi.org/10.21037/tlcr-21-467 


\section{Introduction}

Lung cancer is one of the most prevalent and lethal cancers worldwide (1). The traditional treatments include surgery, chemotherapy, radiotherapy and interventional therapy. The survival of lung cancer patients has dramatically been prolonged in recent years with the availability of targeted therapies, antiangiogenic agents and immune checkpoint inhibitors (ICIs). Meanwhile, technologies for the molecular detection of lung cancer have also advanced rapidly: the detection of single driver genes has evolved to cover combined multi-gene analysis, and whole exome sequencing (WES) has increasingly been applied in the clinical setting. In addition, life support technologies, including ventilators, artificial liver, and artificial kidney as well as extracorporeal membrane oxygenation (ECMO), have further matured, providing powerful forms of life support for patients with various acute and critical diseases.

However, most clinical studies have only enrolled patients with Eastern Cooperative Oncology Group (ECOG) performance status (PS) scores from 0 to 1 , with few patients having PS scores of 2; patients with a PS score of 3 or 4 have been typically excluded. Therefore, due to the lack of high-quality evidence, supportive care is recommended for patients with a PS score of 3 to 4 in the current guidelines. In the real-world, however, approximately $25 \%$ of lung cancer patients present with PS score of 3 or 4 (2) or attain scores between 3 and 4 during the course of treatment. Certain patients with high PS scores can benefit from individualized anti-tumor treatment plus appropriate life-support techniques. In 2017, the Lung Cancer Research Team at the First Affiliated Hospital of Guangzhou Medical College \& Institute of Respiratory Diseases for the first time pioneered the concept of "advanced severe lung cancer" (3) and argued that standardized therapy for chronic obstructive pulmonary disease (COPD) plus anti-tumor therapy can improve both quality of life and prognosis in patients with lung cancer combined with COPD. The authors also found that the early detection of lung cancer driver genes and timely targeted therapy can be successful in treating patients with advanced severe lung adenocarcinoma with a PS score of 4 (4). In 2019, the concept of advanced severe lung cancer was further developed in a featured article (5) that indicated "Advanced severe lung cancer: does not refer to end-stage lung cancer but rather to stage IIIB, IIIC and IV lung cancers with a PS score of 2-4, which can result from a variety of factors related to the disease itself or anti-tumor drugs and which are bighly likely to benefit from the currently available systemic anti-tumor therapies".

In recent years, with the advances in lung cancer diagnosis and treatment techniques and life support technologies, more clinical studies have enrolled patients with a PS score of 2, and some real-world studies have enrolled patients with PS scores of 3-4. Even for patients with early-stage lung cancer, studies have shown that patients with poor PS scores and co-morbidities have a reduced chance of undergoing surgery and an increased mortality rate (6); nevertheless, survival benefit may still be obtained through surgical modifications combined with individualized and multidisciplinary treatment (7). Therefore, the concept of severe lung cancer should not be limited to advanced lung cancer, but applied to all lung cancer patients. In particular, due to the increase in treatment options as well as substantially prolonged survival, the majority of patients may have a PS score between 2 and 4 for a certain period of time due to a variety of reasons. How to provide timely and reasonable treatment for these lung cancer patients has become a critically important realworld research topic. Therefore, we invited lung cancer experts at home and abroad to consider this issue, and this group has reached the following consensus.

\section{Methods}

This consensus was conceived and developed by 87 experts with significant experience in the field. The expert group was divided into 5 subgroups that formulated questions for each topic. The Population, Intervention, Comparison, and Outcome (PICO) framework was used to facilitate systematic literature review. Various databases including PubMed, EMBASE and the Cochrane Library were systematically searched using key words such as lung cancer, poor PS, comorbidities, complications, adverse events (AEs), chemotherapy, radiotherapy, surgery, interventional therapy, epidermal growth factor receptor (EGFR), anaplastic lymphoma kinase (ALK), targeted therapy, antiangiogenic therapy, ICIs and supportive treatment for relevant articles published between January 2000 and March 2021. Each subgroup responded to related topics and developed key guidelines, which were consolidated into 10 key issues (definition, common causes, benefit of diagnosis and treatments, basic diagnosis and treatment techniques, specific diagnosis and treatment strategies, surgical treatment, radiotherapy, interventional techniques, anti-tumor drugs, and life support techniques). The findings 
were graded based on the Oxford Centre of Evidence-Based Medicine's Levels of Evidence (8). Five of us drafted (C Zhou, S Li, J Liu, Q Chu and L Miao) the manuscript and submitted it to all 5 subgroups for discussion and revision. The drafts were distributed to the entire panel of experts and edited multiple times until each participant finally approved it.

\section{Consensus 1: the concept of severe lung cancer}

Severe lung cancer is a disease in which the patient has a PS score between 2 and 4 in certain stages due to various acute or chronic co-morbidities, the tumor itself, and/or treatment-related AEs but which has a high probability of achieving survival benefit and/or improvement in the PS score after supportive care and anti-tumor treatment on the basis of dynamic and precise testing.

This concept addresses three domains:

\section{Etiology}

Three main factors may cause severe lung cancer: (I) various acute and chronic co-morbidities such as heart failure (HF) and COPD; (II) the tumor itself, which may cause massive cardiac and pleural effusions and obstruction of large airways; and (III) various treatment-related AEs such as severe infection due to chemotherapy-induced myelosuppression, interstitial pneumonia due to tyrosine kinase inhibitor (TKI) use, and severe immune-related adverse reactions.

\section{Targeted populations}

Severe lung cancer is a category distinct from end-stage lung cancer. End-stage lung cancer is referred to patients who do not benefit of any specific treatments except palliative care to alleviate symptoms. However, severe lung cancer not only refers to lung cancer patients with PS scores between 2 and 4 in certain stages but also includes those who would enjoy a survival benefit and/or improvement in the PS score with various techniques and individualized multidisciplinary treatment.

\section{The value of diagnosis and treatment}

Treatments must be of viable benefit when performed in patients with severe lung cancer. The value of treatment depends on the state-of-the-art of modern medical technologies. The combination of three such technologies is particularly important: (I) dynamic and precise detection, which enables the timely identification of patients who may benefit from the treatment; (II) powerful life support technologies, which create suitable conditions for various anti-tumor treatments; and (III) anti-tumor treatments, which should be applied individually and thus are highly effective and safe.

\section{Consensus 2: common causes of severe lung cancer}

Three main factors may cause severe lung cancer: (I) various acute and chronic co-morbidities such as $\mathrm{HF}$ and COPD; (II) the tumor itself, which may cause massive cardiac and pleural effusions and obstruction of large airways; and (III) various treatment-related AEs such as surgical complications, radiation injury, severe infections due to chemotherapy-induced myelosuppression, interstitial pneumonia due to TKI use, and severe immune-related adverse reactions.

\section{Acute and chronic co-morbidities}

It was found that $87.3 \%$ of lung cancer patients had at least one comorbidity and $15.3 \%$ had severe comorbidity scores (9). Nieder et al. reported that lung cancer patients without comorbidities had lower PS scores (10). Another study also showed a positive correlation between a simplified comorbidity score (11) and the PS score (12). A study investigated the survival rate of lung cancer patients with comorbidities was significantly lower than that of patients without comorbidities (13).

\section{Heart failure}

The incidence of HF combined with lung cancer is increasing annually, and patients with HF are in fact more likely to develop cancer $(14,15)$. HF increases the risk of death in all lung cancer patients [hazard ratio $(\mathrm{HR})=1.85$ ]; for patients with early-stage lung cancer, HF decreases the likelihood of undergoing surgery and increases postoperative complications (16).

\section{COPD}

COPD is a risk factor for lung cancer and it is present in $40-70 \%$ of lung cancer patients $(17,18)$. It is reported that $50.2 \%$ of non-small cell lung cancer (NSCLC) patients have COPD (19). One-third of lung cancer patients with 
coexisting COPD are not indicated for surgical treatment due to poor lung function (20). A meta-analysis also showed that coexisting COPD was associated with a lower survival rate and a higher rate of postoperative pulmonary complications in lung cancer patients (21).

\section{Interstitial lung disease (ILD)}

A large amount of epidemiological has confirmed a close relationship between ILD and lung cancer. The risk of lung cancer in ILD is 3.5-7.3 times that in the general population, approximately $10-20 \%$. Fifteen percent of ILD patients may die from lung cancer, and the incidence of ILD at the time of lung cancer diagnosis is between $2.4-10.9 \%$ (22). The incidence of lung cancer in the whole course of idiopathic pulmonary fibrosis (IPF) is greater than 50\% (23). Moreover, lung cancer is associated with increased mortality in patients with IPF (24). Among IPF patients who undergo surgery for lung cancer, postoperative acute exacerbation of IPF is reported to occur in approximately $20 \%$, with an associated mortality of about $50 \%(25-28)$. The 5 -year survival rate of stage IA lung cancer patients with ILD was significantly lower than that of those without ILD (54.2\% vs. $88.3 \%, \mathrm{P}<0.0001)(29)$. In patients with lung cancer and ILD, various anti-cancer treatments may also induce acute exacerbation of ILD (22).

\section{Obesity}

Abdominal obesity is a risk factor for lung cancer (30). In the obese population, the presence of adipose tissue around the thorax, abdomen and viscera reduces lung volume and impairs airway stability $(31,32)$. Obese patients have difficulties in coughing up phlegm and turning over in bed, leading to increased perioperative and postoperative complications, and morbid obesity is a risk factor for mortality $(33,34)$.

\section{Lung cancer itself}

Lung cancer itself can lead to a variety of acute and critical complications that require urgent management, and timely management of these complications improve patients' quality of life and PS scores (35).

\section{Pleural effusion}

Pleural effusion occurs in $40 \%$ of lung cancer patients, and malignant pleural effusion is a poor prognostic factor $(36,37)$. The PS score markedly increases as the severity of pleural effusion increases (38). A poor PS score is also a risk factor for poor prognosis in patients with pleural effusion (39).

\section{Pericardial effusion}

Pericardial effusion occurs in approximately $3 \%$ of lung cancer patients (40), and more than one-third of the cases of malignant pericardial effusions are caused by lung cancer $(41,42)$. Pericardial effusion is a risk factor for decreased survival in lung cancer $(43,44)$. Neoplastic pericardial effusion (NPE) patients with PS $\geq 2$ have a worse prognosis (45).

\section{Airway stenosis}

Tracheal or bronchial proximal stenoses occurs as a complication in $20-30 \%$ of lung cancers, resulting in dyspnea, a poor PS score and poor prognosis $(35,46)$. Resolving airway obstruction can rapidly improve the clinical condition and quality of life and decrease PS scores $(47,48)$.

\section{Venous thromboembolism (VTE)}

VTE, including lower extremity deep vein thrombosis and pulmonary embolism, occurs in $13.9 \%$ of lung cancer patients (49). Cancer is a risk factor for VTE, with an HR of 4.7 (50). VTE is 20 times more common in lung cancer patients than in people without cancer. VTE is also a significant cause of death in patients with lung cancer (51).

\section{Treatment-related AEs}

Surgery, radiotherapy, chemotherapy, targeted therapy, antivascular therapy, and immunotherapy are often associated with serious AEs will worsen PS scores; however, timely management of these AEs may improve the PS score.

\section{Postoperative lung injury}

A meta-analysis revealed that the total incidence of postoperative lung injury was $4.3 \%$ for thoracic surgery, and the overall attributable mortality for postoperative lung injury was up to $26.5 \%$; the 1-year survival rate was found to be significantly lower in patients with lung injury (52).

\section{Cerebral radiation necrosis}

Retrospective studies have reported that in patients with metastatic brain tumors, cerebral radiation necrosis occurred in $4.7 \%$ to $9.2 \%$ of patients undergoing stereotactic radiotherapy, at radiotherapy doses ranging from 18 to 30 Gy (53). The incidence of cerebral radiation necrosis was 
$25-50 \%$ in patients treated with brachytherapy (54).

\section{Chemotherapy-induced neutropenia accompanied by infection}

Neutropenia is a common hematologic toxicity induced by chemotherapeutic agents. Prolonged neutropenia is associated with increased risk of infection (55). The reported rate of mortality is $11.2 \%$ in lung cancer patients with febrile neutropenia (56).

\section{Bleeding caused by angiogenesis inhibitors}

In a meta-analysis including 12,617 patients with a variety of solid tumors, the incidence of hemorrhage was $30.4 \%$ after bevacizumab use, with $3.5 \%$ being high grade; the risk for fatal bleeding was only $0.8 \%$, but was significantly elevated in lung cancer [relative risk $(\mathrm{RR})=5.02](57)$.

\section{TKI-associated ILD}

A meta-analysis showed that the incidence of all-grade and high-grade ( $\geq$ grade 3 ) ILD associated with EGFRTKIs was $1.6 \%$ and $0.9 \%$, with a mortality of $13.0 \%$ (58). In another meta-analysis, the incidence of all-grade and high-grade ILD associated with ALK-TKIs was 2.14\% and $1.33 \%$, respectively (59).

\section{Checkpoint inhibitor-related pneumonitis (CIP)}

The incidence of CIP was found to be $3-5 \%$ in clinical studies and $7-13 \%$ in real-world studies (60), with a casefatality rate of $12.8-22.7 \%$ (61-63). The severity of CIP is positively correlated with the PS score (64).

\section{Consensus 3: benefits of the diagnosis of and treatment for severe lung cancer}

Severe lung cancer is different from end-stage lung cancer, and the benefits of clinical treatment is worthy of emphasis. When a PS score is placed between 2 and 4, it should be aware that the PS score is stage-specific, "reversible", or "fluctuating". It should also be evident whether the survival benefit can be obtained in severe lung cancer patients through individualized treatment with the currently available techniques (recommendation category: B; level of evidence: $2 \mathrm{a}$ ).

\section{Reversibility of the PS score}

One study investigated the efficacy and feasibility of gefitinib for patients with advanced NSCLC and found the
PS improvement rate to be $79 \%$; in particular, $68 \%$ of the 22 patients improved from PS 3-4 at baseline to PS 0 or 1 (65). A retrospective study analyzing chemotherapy for advanced NSCLC with a PS score $\geq 2$ showed that $45.26 \%$ of the patients had improved PS scores (66). In patients with massive pleural and pericardial effusion, drainage rapidly relieved the symptoms $(39,67,68)$. In patients with major airway obstruction, timely and effective local treatment immediately alleviated the symptoms related to the airway obstruction $(47,48)$. Thus, the PS score is reversible, and the key lies in resolving the immediate causes [e.g., oncologic emergencies (69)] of the poor PS score.

\section{PS scores fluctuation}

In patients with lung cancer accompanied by chronic disease(s), the recurrence or exacerbation of the underlying disease may lead to fluctuations in the PS score. A study of 882 lung cancer patients showed a negative correlation between PS score and increasing severity of the comorbidities (70). A previous study analyzing NSCLC with a PS $\geq 2$ showed that the score was improved in $91.7 \%$ of the patients after aggressive ventilatory support and management of the vital organ-centered comorbidities (3). In another study, $40 \%$ of 70 lung cancer patients with a PS score of 2-3 had underlying disease of the lungs; after treatment with anlotinib plus S-1, the PS score decreased and displayed stage-specific fluctuations (71).

\section{Consensus 4: basic diagnosis and treatment techniques for severe lung cancer}

The relative value of severe lung cancer treatment depends on the advances made in modern medical technology. The combination of three clinical technologies is particularly important: (I) dynamic and precise detection, which enables the timely identification of patients who may benefit from the treatment; (II) powerful life support technologies, which create the conditions needed for various anti-tumor treatments; and (III) individualized anti-tumor treatments that are highly effective and safe.

\section{Dynamic and precise detection (recommendation category: A; level of evidence: $1 b$ )}

The past decades have witnessed the transition of lung cancer treatment from traditional chemotherapy to precision therapy. Targeted therapies are preferred for 
patients with driver gene-positive tumors; for patients without driver genes, non-targeted therapy biomarkers should be detected in order to identify those patients in which there is potential benefit. Clinicians should test tissue specimens whenever possible. However, tissue specimens may not be obtainable for genotyping in some patients, in which case liquid biopsy should be performed. Tumors are highly heterogeneous, and puncture specimens can only reflect the localized lesions. Moreover, the genetic status and tumor status may change after antitumor therapy, and subsequent treatments based only on the results of the initial specimen may be inaccurate $(72,73)$. Therefore, the pathological type and genetic status should be dynamically examined throughout the course of treatment. Liquid biopsy has the advantages of easy access, low invasiveness, and good repeatability, all of which enable dynamic detection $(74,75)$.

\section{Life support techniques (recommendation: B; level of evidence: $2 a$ )}

Patients with severe lung cancer should be given appropriate life support so as to enable subsequent anti-tumor treatment. Many novel life support technologies including non-invasive/invasive ventilation, liver replacement, kidney replacement, and ECMO have saved the lives for many of critically ill patients.

\section{Anti-tumor treatments (recommendation: B; level of evidence: $2 a$ )}

Anti-tumor treatment and life support do not conflict with each other. Clinicians should treat both symptoms and their causes: when actively managing complications and comorbidities, the most appropriate anti-tumor treatment needs to be identified. At present, anti-tumor treatments include surgery, chemotherapy, radiotherapy, interventional therapy, angiogenesis inhibitors, targeted therapy and immunotherapy or any combinations of them. The antitumor treatments should be used flexibly, and those drugs having the highest efficacy and lowest toxicity are obviously preferred.

\section{Consensus 5: specific diagnostic and treatment strategies for severe lung cancer}

There is currently no well-controlled, prospective clinical evidence available for determining the optimal treatments for severe lung cancer; however, indirect data from research on patients with PS 0-1 may be useful for making decisions. Flexible and individualized treatment strategies can be adopted, which include but are not limited to: "treatment of both the lung cancer and other lung diseases", "selection of treatment approaches according to the PS score", "escalation and de-escalation of anti-tumor drugs", "dynamic and precise detection", "smart combinations for increasing efficacy and reducing toxicity" and "multidisciplinary participation, and individualized and comprehensive treatment".

\section{Treatment of both lung cancer and other lung diseases (recommendation category: $B$; level of evidence: $2 a$ )}

During the treatment of lung cancer, the management of other acute and chronic respiratory diseases such as COPD, ILD, pulmonary embolism, and lung infections must not be neglected. Zhang et al. found the overall diagnostic rate of COPD was only $7.1 \%$ (50 of 705 lung cancer patients meeting the diagnosis criteria of COPD), and the treatment for stable and acute exacerbation of COPD was $27.1 \%$ and $46.8 \%$, respectively (76). Poor management of COPD can lead to a decrease in patients' quality of life, decrease in therapeutic efficiency, and worsening of the prognosis. In a previous study, patients receiving anti-tumor therapy plus proper COPD management had significantly longer progression-free survival (PFS) and overall survival (OS) compared to those treated with anti-tumor therapy alone (77). In a real-world study in the Republic of Korea that retrieved clinical information from a medical information system, 113 of 8,014 NSCLC patients were found to have unsuspected pulmonary emboli (PE), and the results showed that the mortality rate was 4.1 times higher in those patients who did not receive anticoagulation therapy than in those who did (78). The treatment protocols for lung cancer are selected based on the PS score, which can be directly affected by pulmonary comorbidities and complications. Therefore, controlling for comorbidities or complications as well as treating lung cancer is important, and such findings represent the primary and secondary aspects of the "contradiction" involved in the treatment of severe lung cancer. While the primary aspect is undoubtedly important, the primary and secondary aspects can be impact each other. Therefore, the management of severe lung cancer requires that attention be paid to both the primary and secondary aspects (i.e., both the lung cancer 
and that other lung diseases require treatment).

\section{Dynamic and precise detection (recommendation category: A; level of evidence: 1 b)}

At present, targeted therapy is mainly used in driver genepositive patients; the use of anti-vascular therapy is typically avoided in high-risk patients; immunotherapy is not a precise treatment as it is preferred only in certain select populations and should be avoided in the high-risk groups; and chemotherapy has only a limited tissue-specific effect. Dynamic and precise detection for a timely identification of targets is particularly important for patients with severe lung cancer over their lifetimes.

\section{Chemotherapy targets (recommendation category: C; level of evidence: $2 \mathrm{a}$ )}

Many studies have been devoted to identifying predictors of chemotherapy efficacy. It is reported that high excision repair cross complementing-group 1 (ERCC1) expression is associated with decreased efficacy of platinum agents (79), high ribonucleotide reductase subunit 1 (RRM1) expression is associated with poor efficacy of gemcitabine (80), increased thymidylate synthase (TS) expression is associated with poor efficacy of pemetrexed (81), and class III beta-tubulin (TUBB3) is associated with resistance to toxoids (82). However, currently these biomarkers are not considered clinically useful and thus require further investigation.

\section{Targets used in targeted therapies (recommendation category: A; level of evidence: $1 \mathrm{a})$}

Currently, the driver genes routinely tested in clinical laboratories include EGFR, ALK, ROS1, KRAS, HER-2, BRAF V600, RET fusion, MET amplification, and MET14 exon skipping. Targeted therapy requires the detection of specific targets, and only precisely targeted therapy can work quickly and effectively. However, drug resistance can occur during targeted therapy, and a repeated detection of the relevant targets is needed to determine the mechanisms of resistance. For example, approximately $50 \%$ of patients with EGFR-sensitive mutations treated with first- and second-generation EGFR-TKIs develop T790M mutations, which can be effectively treated with third-generation TKIs (83).

\section{Targets of anti-angiogenesis therapies} (recommendation category: $\mathrm{C}$; level of evidence: 1a) Currently, single-target drugs include bevacizumab
(VEGF-A) and ramucirumab (VEGFR-2), multi-target (targeting VEGFR, PDGFR, FGFR, RAF, etc.) smallmolecule TKIs include anlotinib, apatinib, sunitinib, sorafenib, nintedanib, and fruquintinib, and pan-target drugs include vascular endothelial inhibitors (84). A phase II/III clinical study involving 878 NSCLC patients randomly assigned carboplatin + paclitaxel $(\mathrm{PC})$ or $\mathrm{PC}+$ bevacizumab (BPC). The results showed that patients with high baseline VEGF-A had higher response to BPC than those with PC; and bevacizumab was beneficial to PFS in patients with low baseline intercellular adhesion molecule (ICAM) (85). Another study to investigate the biomarkers of bevacizumab combined with chemotherapy for NSCLC patients showed that VEGF-A was not associated with response to bevacizumab, but low VEGF-A was associated with favorable PFS and OS (86). However, there are still no reliable predictive biomarker for the efficacy of antivascular therapy. The use of these drugs has no clear requirement for the PS score, and the current suggested treatment strategy is to avoid contraindications such as patients at high risk of bleeding, in an active thrombotic phase, having high risk pulmonary cavity, and/or uncontrollable hypertension.

\section{Immunotherapy targets (recommendation category: B;} level of evidence: 1a)

Patients with high levels of PD-L1 expression are reportedly more likely to respond to immunotherapy (87). Le et al. demonstrated a positive correlation between efficacy and mismatch repair-deficient (dMMR)/ microsatellite-instability-high (MSI-H) (88). In 2017, the US FDA approved pembrolizumab for any solid tumor with a specific genetic biomarker for MSI-H or dMMR (89). The CheckMate 227 study showed tumor mutational burden (TMB) was a positive marker (90), but the KEYNOTE-189 and KEYNOTE-021 studies presented in 2019 World Conference on Lung Cancer (WCLC) reported that TMB did not predict the efficacy of immunotherapy combined with chemotherapy. POLE/POLD1 mutations are reportedly associated with a good efficacy of ICI (91). In addition, tumor neoantigens, tumor infiltrating lymphocytes, transcriptional features of the immune response and the microbiome are all reportedly related to the efficacy of immunotherapy (92). Recently, multiple studies have focused on factors related to hyperprogression, such as MDM2/MDM4 amplification, EGFR amplification and genes at $11 \mathrm{q} 13$, such as cyclin D1 (CCND1), and fibroblast growth factor $(F G F)$ 3, 4, 19 (93-95). 


\section{PS score and escalation/de-escalation strategies (recommendation category: $B$; level of evidence: $3 a$ )}

The efficacy and toxicity of different anti-tumor drugs vary. For patients with severe lung cancer, a low-toxicity, highefficacy regimen (or even a combination regimen)—but not a first-line standard regimen - can be used at first, with the aim of breaking the vicious cycle between the tumor and the severe disease status, which is known as an "escalation strategy"; in contrast, once the disease status has improved, a more tolerable standard anti-tumor regimen can be applied according to the patient's specific situation and needs, known as a "de-escalation strategy". The PS score is used as a stratifying factor for guiding treatment in all of the currently available guidelines on lung cancer. Patients with severe lung cancer typically have a poor PS score. However, PS scores fluctuate and are reversible. Therefore, patients with severe lung cancer should have their treatment options chosen according to their PS scores. For patients with a poor PS score, it is important to identify the etiology and actively manage any comorbidities and complications. A low-toxicity and high-efficacy anti-tumor regimen can be applied at first, and then the anti-tumor regimen may be adjusted once the PS score has improved. Thus, the PS score-based escalation and de-escalation strategy refers to the strategy of escalating or deescalating the use of antitumor regimen according to the efficacies and toxicities of anti-tumor drugs in patients with different PS scores on the basis of the treatment protocols recommended by the currently available guidelines. A recent study has also suggested that for patients with small cell lung cancer (SCLC) with poor PS scores, the reasons for the poor scores should be actively sought out and chemotherapy utilized at first, followed by immunotherapy once the PS score has improved (96).

Optimizing combination treatments for increasing efficacy and reducing toxicity (recommendation category: B; level of evidence: 1 a)

Chemotherapy, radiotherapy, anti-vascular therapy, targeted therapy, and immunotherapy have different anti-tumor mechanisms, and all of them have their own advantages and adverse effects. Appropriate combination therapy can achieve better efficacy while reducing adverse effects. In the global phase III IMpower150 study, immunotherapy combined with anti-vascular therapy + chemotherapy was more effective than anti-vascular therapy + chemotherapy in treating NSCLC patients with a high tumor burden, liver metastases, and positive driver genes (EGFR/ $A L K)$, along with fewer fatal AE (i.e., immune-associated pneumonia) (97). In another phase III trial (NEJ026) comparing the effectiveness and safety of erlotinib plus bevacizumab $v s$. erlotinib alone, the combination group displayed better efficacy, and no interstitial pneumonia was noted in the combination group (98). The combinations of antiangiogenic agents with EGFR-TKI increase the antitumor efficacy and overcome the resistance of EGFRTKI. A recent study compared the efficacy and safety of a regimen of combining anlotinib and S-1 vs. anlotinib monotherapy in treating patients with advanced squamous cell lung cancer having a poor PS scores [2-3]. The combination group had significantly longer OS, and there was no grade 3 or higher toxicity (71).

\section{Multidisciplinary participation, and individualized and comprehensive treatment (recommendation category: A; level of evidence: $1 b$ )}

Patients with severe lung cancer need the participation of different disciplines, mutual communication and collaboration, and the development of individualized and comprehensive treatment. In addition, patients' active cooperation is also very important. Bossert et al. interviewed 15 patients with stage IV lung cancer with comorbidities and found that they believed that patients played an active role in multidisciplinary treatment (99). A prospective study showed that compared with conventional preoperative pulmonary rehabilitation, preoperative multidisciplinary pulmonary rehabilitation therapy can reduce postoperative complication rate $(48.3 \%$ vs. $28.6 \%, \mathrm{P}=0.2428)(100)$. The determination of the cause of severe lung cancer, the choice of anti-tumor treatment, the treatment of comorbidities and complications, and the implementation of life support technology all require multidisciplinary participation. Multiple studies have shown multidisciplinary team (MDT) approach in the treatment of lung cancer can improve quality of life and OS rate of patients (101-103).

\section{Consensus 6: application of surgery in patients with severe lung cancer}

Patients with early-stage lung cancer can also have various other underlying diseases such as cardiopulmonary comorbidities, which limit the feasibility of conventional surgery. For patients with early-stage severe lung cancer 
and various comorbidities, a variety of sophisticated modern testing, examination, treatment, and life support technologies can be used to ensure successful preoperative assessment, intraoperative protection, and postoperative support; meanwhile, a MDT can be engaged to enable optimal coordination and cooperation among the related departments including thoracic surgery, anesthesiology, respiratory and critical care, and nutrition (recommendation category: B; level of evidence: 2 a).

The most common reason for surgery in patients with early-stage severe lung cancer is lung cancer accompanied by COPD. Here we describe the application of surgery to such patients.

\section{Pre-operative assessment}

\section{Pulmonary function tests}

Pulmonary function tests are of critical importance prior to lung surgery. The widely recognized contraindications to surgery include: a predicted baseline forced expiratory volume in one second $\left(\mathrm{FEV}_{1}\right)$ of less than $40 \%$; a predicted postoperative $\mathrm{FEV}_{1}$ value of less than $30 \%$; and a predicted diffusion capacity of the lung for carbon monoxide (DLCO) of less than $40 \%$.

Ventilation test: pulmonary ventilation is a dynamic temporal process involving the entry of air into and carbon dioxide exit from the alveoli. The commonly used indicators include ventilation at rest, alveolar ventilation, maximal voluntary ventilation (MVV), timed vital capacity, and certain flow rate indicators. Clinically, COPD patients mostly present with obstructive ventilation dysfunction [i.e., decreased flow rate (the $\mathrm{FEV}_{1} / \mathrm{FVC}$ ratio)], which can be classified as follows: mild COPD (predicted $\mathrm{FEV}_{1} /$ $\mathrm{FVC}<70 \%, \mathrm{FEV}_{1} \geq 80 \%$ ); predicted moderate COPD (predicted $\mathrm{FEV}_{1} / \mathrm{FVC}<70 \%, 50 \% \leq \mathrm{FEV}_{1}<80 \%$ ); severe $\mathrm{COPD}$ (predicted $\mathrm{FEV}_{1} / \mathrm{FVC}<70 \%, 30 \% \leq \mathrm{FEV}_{1}<50 \%$ ); and very severe COPD (predicted $\mathrm{FEV}_{1} / \mathrm{FVC}<70 \%$, $\left.\mathrm{FEV}_{1}<30 \%\right)$. Lung diffusion testing measures how well the lungs exchange gases. As a measure of alveolarcapillary membrane functional efficiency, it is important for detecting early lung and airway lesions, assessing the severity of the disease and forming its prognosis, evaluating the efficacy of drugs or other treatments, and identifying the cause of the dyspnea. Locating the lesions and assessing the functional operability are highly valuable. The normal values for DLCO are $28.84 \pm 4.84 \mathrm{~mL} / \mathrm{mmHg} / \mathrm{min}$ for men and $22.13 \pm 3.09 \mathrm{~mL} / \mathrm{mmHg} / \mathrm{min}$ for women. Diffusion impairment can be divided into three levels based on severity: mild (predicted $\geq 60 \%$ but $<80 \%$ ), moderate (predicted $\geq 40 \%$ but $<60 \%$ ), and severe (predicted $<40 \%$ ).

In general, patients with mild ventilatory dysfunction can tolerate single-lobe resection; for patients with moderate to severe ventilatory dysfunction, the feasibility of lobectomy should be assessed in a comprehensive manner; for patients with very severe ventilatory dysfunction or those with accompanying moderate or severe diffusion dysfunction, surgery requires extreme caution.

\section{Breathing and nutritional support}

Breathing functionality and nutritional support are also important and necessary. Perioperative physical therapy can enhance postoperative respiratory function recovery. Also, a low body mass index (BMI) is associated with postoperative negative nitrogen balance. There is evidence that postoperative complications are markedly increased in patients with a BMI of below $14 \mathrm{~kg} / \mathrm{m}^{2}$ (104). A randomized controlled study showed enteral nutrition plus accelerated rehabilitation reduced postoperative complications and improved postoperative recovery, compared with conventional nutrition therapy (105).

\section{Pharmacotherapy}

All lung cancer patients with accompanying COPD can receive standardized COPD treatment for at least 1 week in order to improve their lung function. Nebulizers and oral medications are preferred for pharmacotherapy. The most commonly used nebulized medications include hormones, acetylcholine receptor antagonists and $\beta 2$-agonists; leukotriene receptor antagonists or theophylline may also be used; and if necessary, antibiotics should be used to control chronic inflammation.

\section{Blood gas analysis}

The arterial partial pressures of oxygen and carbon dioxide are also important measures of postoperative complications, especially in patients who are unable to cooperate with ventilation and diffusion capacity tests. Carbon dioxide retention is a more important indicator than hypoxemia.

\section{Cardiac color ultrasound}

In addition to the assessment of cardiac structure and valve function, cardiac color ultrasound also affords the ejection fraction (EF) value $(50 \%$ or higher allows a safe surgery). The presence of pulmonary hypertension is also a contraindication to pulmonary surgery. 


\section{Pulmonary ventilation/perfusion scan}

Pulmonary perfusion imaging reveals any blood perfusion in lung tissues, and the commonly used radiopharmaceutical for this purpose is Tc-labeled macroalbumin (Tc-MAA). Pulmonary ventilation imaging reflects the gas filling the airways and alveoli. Pulmonary ventilation/perfusion scan can assist the assessment of split/lobar lung function, thus determining the local function of the resected lung tissue and predicting the residual lung function after the surgery.

\section{Pre-operative assessment of functional exercise capacity}

In clinical practice, the stair-climbing test is obviously not an objective way to measure the cardiopulmonary function and surgical tolerance in surgical patients. In contrast, the six-minute walk test (6MWT) has been used to evaluate the efficacy of therapeutic interventions in patients with moderate to severe cardiopulmonary disease and to measure the functional status of patients. It is widely recognized as a simple, and reliable tool for evaluating cardiopulmonary function. 6MWT was officially released by the American Thoracic Society (ATS) in 2002, along with a comprehensive guideline (106). The incidences of pulmonary complications (36.9\%), atrial fibrillation (11.5\%), and blood transfusion (9.0\%) significantly increased after lobectomy in patients, with a $6 \mathrm{MWT}<500 \mathrm{~m}$, and the average hospital stay was also up to 7 days (6 days in patients with a 6MWT $>500 \mathrm{~m}$ ) (107). Similarly, the risk of pulmonary complications increased in patients with a $6 \mathrm{MWT}<300 \mathrm{~m}$ (108).

\section{Three-dimensional (3D) reconstruction}

$3 \mathrm{D}$ reconstruction of the lungs has been increasingly used for pulmonary surgery planning, especially during sublobar resections. Based on preoperative thin-section computed tomography (CT), 3D reconstruction of lung segments, pulmonary vessels, and bronchi can display the microscopic structures in a more intuitive manner (109). In addition, it allows more detailed measurement of the local lung volume, which assists in determining the functional loss after lung surgery.

\section{Intraoperative assurance}

\section{Surgical planning}

Lobectomy vs. sublobectomy: anatomic lobectomy is superior to sublobectomy (segmentectomy or wedge resection) for patients with stage I NSCLC. However, many COPD patients have impaired lung function, which may affect their ability to tolerate the procedure. Compared with lobectomy, sublobectomy may result in a significantly higher rate of local recurrence (110). However, patients who cannot tolerate lobectomy should only undergo sublobar resection.

The selection of potential patients eligible for lobectomy should be based on a series of cardiopulmonary function tests prior to the surgery: (I) no carbon dioxide retention is found upon blood gas tests; (II) echocardiography reveals good cardiac function (with an $\mathrm{EF}>50 \%$ ); (III) pulmonary diffusion capacity must be at least moderate; (IV) preoperative 6MWT should be more than grade 2; and $(\mathrm{V})$ pulmonary ventilation/perfusion scan with $3 \mathrm{D}$ reconstruction of lung tissue is recommended. Preoperative assessment of lung function in the target area is essential for surgical planning and outcome prediction and enables accurate quantitative characterization of ventilation and perfusion capacity at the lobe or even segment level. If the tumor is located in a lobe afflicted with severe emphysema, patients must undergo resection of the lung cancer while simultaneously removing the poorly functioning lung tissue, which in turn improves lung function. This phenomenon is called "the lung volume reduction effect". For example, some patients with poor lung function resulting in shortness of breath can safely undergo pneumonectomy for both lung cancer and lung volume reduction at an acceptable level of risk, and the procedure may even improve their lung capacity $(111,112)$. In another study, upper lobectomy showed a volume reduction effect, suggesting patients with a lower preoperative $\mathrm{FEV}_{1.0} \%$ of predicted had a greater "volume reduction effect" with an increase in $\mathrm{FEV}_{1.0}$ after upper lobectomy (113).

\section{Intraoperative assessment}

Intraoperative hypoxic preconditioning (HPC) or ischemic preconditioning (IPC) is an important component of the intraoperative assessment that is performed to determine the surgical approach. Studies have shown that HPC or IPC can improve the tolerance of cells, tissues, organs, and even the organism itself to subsequent severe hypoxia or ischemia. HPC also improves postoperative oxygenation, enhances lung function recovery, and reduces the length of hospital stay (114). During the surgery, it may be feasible to lower the inhaled oxygen concentration to below $50 \%$ while the operator clamps the pulmonary artery to the target lobe to block the blood supply. Meanwhile, the oxygenation index [the arterial partial oxygen pressure $\left(\mathrm{PaO}_{2}\right)$ /fraction of inspired oxygen $\left(\mathrm{FiO}_{2}\right)$ ] must be $\geq 200$ (with no significant 
change in vital signs) for 10 minutes. Lobectomy is considered safe and feasible if these conditions are met.

\section{Postoperative support}

\section{Lung infection}

Pulmonary infection and pneumonia are major causes of postoperative morbidity and mortality. A Cochrane systematic review of seven randomized controlled trials highlighted the fact that combined corticosteroid/longacting beta 2 agonist (LABA) (fluticasone and salmeterol) increased the risk of pneumonia in COPD patients (115). Treatment of postoperative pulmonary infections begins with pathogenic exam of respiratory samples, followed by assessment for the presence of sepsis and risk factors for multidrug-resistant pathogens. Then, according to the results from a drug sensitivity test and the risk factors, adequate selection of antibiotics and the escalation or deescalation principle is required (116).

\section{Persistent air leaks in the lungs}

Pulmonary air leak is common in COPD patients, which may be explained by the fact that the impaired lung parenchyma and the diminished elastic recoil of the lungs after emphysema delay the healing of lung tissue (117). Lobectomy is associated with a higher rate of air leak than sublobectomy, which may be due to the fact that radical anatomical resection results in larger soft-tissue damage and a significantly longer healing time. Thus, in addition to intensive nutritional support, continuous negative-pressure suction and intrathoracic injection of adhesives are also critically important treatments after the surgery.

\section{Atelectasis}

Postoperative atelectasis can be treated with airway clearance techniques, including postural drainage and coughing, and bronchial suctioning using bronchoscopy or tracheal catheter as needed (118).

\section{Consensus 7: application of radiotherapy techniques in patients with severe lung cancer}

Radiotherapy is indicated in all stages of lung cancer. For patients with severe lung cancer, radiotherapy may play the following three roles: (I) radical radiotherapy for patients with severe disease who cannot tolerate surgery even in the early stage; (II) combination of precise radiotherapy with drugs in patients with locally advanced severe lung cancer to achieve the same purpose as radical treatment; and (III) palliative radiotherapy for patients with advanced severe lung cancer; its application in special areas can rapidly improve the severe symptoms, and its combinations with medical treatments can also benefit patients (recommendation category: B; level of evidence: $2 \mathrm{a}$ ).

\section{Radical radiotherapy for patients with severe disease who cannot tolerate surgery even in the early stages}

Surgery remains the standard treatment modality for earlystage NSCLC. However, radiotherapy is also an effective treatment for those who cannot tolerate surgery for various reasons (e.g., advanced age, poor lung function, poor PS score, or the co-existence of other serious systemic diseases). One study found that stereotactic body radiation therapy (SBRT) and sublobar resection had similar 5-year survival rates in patients with stage I NSCLC who could not tolerate lobectomy (40.4\% vs. 55.6\%, $\mathrm{P}=0.124$ ) (119). SBRT or stereotactic ablative radiotherapy (SABR) is significantly more effective than conventional fractionated radiotherapy, with a 3-year local control rate of $73-91 \%$ and a 3-year OS rate of $43-60 \%$. SBRT/SABR is the current standard radiotherapy modality for early-stage NSCLC. Previous clinical studies have shown that SBRT with a biologically equivalent dose (BED) of $\geq 100-105$ Gy achieves better local control and OS, and a higher dose is associated with better outcomes. Therefore, the recommended dose of SBRT is to achieve BED of $\geq 100 \mathrm{~Gy}$; however, the organ-threatening dose and the tolerability of patients should be strictly evaluated (120-122).

Radical resection is also a standard treatment for earlystage SCLC $\left(\mathrm{T}_{1-2} \mathrm{~N}_{0} \mathrm{M}_{0}\right)$. As the case for NSCLC, SBRT/ SABR is also recommended for SCLC patients who cannot tolerate surgical treatment for some reason (e.g., advanced age, poor lung function, poor PS score, or the co-existence of other serious systemic diseases), with a recommended irradiation dose of BED $\geq 100$ Gy, which can achieve a 3 -year local control rate of $96.1 \%$ and a 3 -year OS rate of $34.0 \%(123-125)$.

\section{Radiotherapy for patients with locally advanced severe lung cancer}

Durvalumab as consolidative immunotherapy following concurrent chemoradiotherapy (cCRT) is the standard treatment for patients with unresectable advanced NSCLC. However, cCRT may result in aggravated the toxicities 
and is thus mainly applicable to patients with a PS score of $0-1$, while patients with poorer PS scores [2-4] often cannot tolerate cCRT. It was found that the incidence of grade 3-4 toxicities was significantly lower in the sequential chemoradiotherapy group than the cCRT group (4\% vs. $18 \%)$; although the sequential chemoradiotherapy group had slightly inferior efficacy compared with the cCRT group, and the 3-year local control rate still reached $28.1 \%$ and the 3 -year OS rate reached $18.1 \%$. Therefore, sequential chemoradiotherapy or radiotherapy alone can be considered for patients with poor PS scores $(126,127)$. EGFR-TKIs have demonstrated superior efficacy and safety over chemotherapy in advanced NSCLC patients with EGFR-sensitive mutations. The combinations of targeted therapy with radiotherapy are being explored in patients with EGFR-positive locally advanced NSCLC. A retrospective study presented at the 2020 American Society of Clinical Oncology (ASCO) Annual Meeting showed that, for EGFR-positive locally advanced NSCLC, the median PFS was 21.6 months and the median OS was 67.4 months in the EGFR-TKIs + concurrent radiotherapy group, the median PFS was 16.2 months in the radiotherapy + sequential EGFR-TKIs group, and the median PFS was 12.7 months in the radiotherapy alone group (128).

Compared with conventional cCRT, radiotherapy combined with EGFR-TKIs is expected to improve the outcomes of EGFR-positive unresectable, locally advanced NSCLC. In terms of prospective studies, a phase III trial on osimertinib (LAURA) and a phase II study on icotinib (NCT03396185) are underway and the results are reportedly promising. Given the superior safety profile of targeted therapy, this treatment modality is particularly suitable for patients with severe lung cancer with poor PS scores.

\section{Palliative radiotherapy in patients with advanced severe lung cancer}

Systemic therapy-based multidisciplinary treatment is the standard treatment for advanced lung cancer. For patients with severe lung cancer with poor PS scores, the values of radiotherapy mainly lie in: (I) palliative radiotherapy for local lesions to improve patients' PS scores; and (II) increasing the control rate of local lesions and improving survival when systemic treatment is effective.

The central nervous system (CNS) is a common metastasis site of lung cancer, especially for patients with positive driver gene mutations. Brain metastases are a serious threat to patients' lives. Radiotherapy for brain metastases helps to control lesions, improve symptoms, and prolong survival. The most appropriate radiotherapy technique should be selected according to the pathological type of the malignancy, the number and extent of the brain metastases, and the distance to the threaten organs. Compared with whole-brain radiotherapy (WBRT), stereotactic radiosurgery (SRS) has the characteristics of a more precise localization, higher local dose, less damage to surrounding tissues, and less serious side effects, and thus in the preferred brain radiotherapy technique for patients with severe NSCLC. In particular, for patients with small brain metastases (maximal diameter of up to $3 \mathrm{~cm}$ ) and of limited number $(\leq 4)$, SRS is preferred. The JLGK0901 study found that SRS alone in the treatment of patients with 5-10 metastases is equivalent to 2-4 metastases (129). Compared with SRS alone, the combination of SRS and WBRT can reduce the probability of intracranial recurrence, but it has no benefit in improving long-term survival and increases the risk of cognitive impairment (130). Therefore, for patients with a limited number of metastases $(\leq 10)$, SRS alone is the preferred local treatment. If the lesions are adjacent to vital centers, hypofractionated SRS may be considered to further reduce the side effects $(131,132)$. SCLC has a high propensity to metastasize into the brain due to certain biological features, and therefore WBRT is recommended for SCLC patients. For those with oligometastases, SRS used as boost irradiation on the basis of WBRT can be considered to improve local control rate $(133,134)$. In addition, brain necrosis and edema after radiotherapy for brain metastases, especially SRS, are also clinical issues. $\mathrm{Li}$ et al. reported that the biologically effective doses and gross tumor volume $(\mathrm{BED} \times \mathrm{GTV})$ can effectively indicate the time interval and edema range of brain necrosis after radiotherapy, which can be used to guide the use of bevacizumab (135).

Meningeal metastatic carcinoma is the spread of tumor cells in the circulating cerebrospinal fluid, and deposits form on the surface of the meninges and nerve roots, resulting in the absorption of cerebrospinal fluid. This causes a series of serious clinical symptoms, such as high intracranial pressure, mental change, abnormal gait, cranial nerve paralysis and secondary epilepsy. Meningeal metastases are often more threatening to patients' lives than brain parenchymal metastases. The diagnosis of meningeal metastasis depends on magnetic resonance imaging (MRI) and cerebrospinal fluid cytology. WBRT can improve the symptoms of patients with meningeal metastatic carcinoma and even afford survival benefits to some patients (136). 
Local high-dose radiotherapy can also be considered for mass meningeal metastases. A ventricle-abdominal shunt can effectively alleviate the symptoms of the high intracranial pressure caused by meningeal metastatic carcinoma and promotes the smooth completion of WBRT. Intrathecal chemotherapy (e.g., pemetrexed) can solve the problem of the low intracranial blood drug concentration caused by peripheral intravenous administration due to the obstruction of the blood-brain barrier. In addition, Ommaya capsule implantation can effectively increase the blood concentration of cerebrospinal fluid and also provides a convenient, minimally invasive and continuous administration route for regular intrathecal chemotherapy. Palliative radiotherapy for other sites includes but is not limited to: (I) treatment of bone metastases, where it helps to relieve local pain and lower the incidence of bone related events such as pathological fractures and paraplegia; (II) treatment of hemoptysis in patients with central lung cancer accompanied by poorly controlled hemoptysis, palliative radiotherapy can help to stop hemorrhaging after the site of bleeding is clearly identified by endoscopic or radiological techniques; and (III) treatment of superior vena cava (SVC) syndrome: SCLC often presents as a centrally located mass that is often combined with SVC syndrome, which decreases patient quality of life. Hyperfractionated radiotherapy can be used to rapidly relieve the obstruction and alleviate the relevant symptoms.

When the tumor is controlled after systemic therapy, local radiotherapy for residual primary and/or metastatic lesions may achieve prolonged disease control and survival (137-139). Results of a phase II study in a Chinese NSCLC population published in 2015 showed that patients who received palliative radiotherapy for primary lesions in the chest had a 1-year local control rate of $78.8 \%$, a median PFS of 9.0 months, and a median OS of 13.0 months (140). Palliative radiotherapy for metastases is also expected to prolong survival in patients with NSCLC. The results of a phase II study published in 7 Clin Oncol in 2019 showed that palliative radiotherapy for metastases significantly prolonged PFS (14.2 vs. 4.4 months, $\mathrm{P}=0.022)$ and $\mathrm{OS}$ (41.2 vs. 17.0 months, $\mathrm{P}=0.017$ ) in NSCLC patients when systemic therapy was effective $(141,142)$.

\section{Consensus 8: application of interventional techniques in patients with severe lung cancer}

Interventional techniques for lung tumors can be applied via the airway, chest wall, or blood vessels. Lung cancer patients can undergo a variety of acute and critical conditions such as large airway obstruction, acute pulmonary embolism, and hemoptysis. Proper interventional techniques can rapidly alleviate or control these clinical symptoms and improve PS scores, thus making other anti-tumor therapies possible and even achieving a cure for some specific types of lung cancers $(143,144)$. (Recommendation category: B; level of evidence: $2 \mathrm{a})$.

\section{Interventional treatment of central airway obstruction (CAO)}

Clinically, lung cancer-related CAO (which can be divided into endogenous, exogenous, and mixed types, depending on the tumor cell infiltration of the airway) can range from asymptomatic to life-threatening, and interventional therapy can rapidly alleviate symptoms and create opportunities for subsequent treatment in critically ill patients. The endogenous type can be treated by mechanical resection or ablation (including electrocoagulation/electrocision/loop electroexcision procedure, laser, argon plasma coagulation, photodynamic therapy, and cryotherapy). For the exogenous type, intervention techniques include dilatation and stent implantation. In certain patients, combinations of several different types of intervention are often used. In particular, the interventional techniques may be applied in combination with brachytherapy to achieve long-term benefit. With the participation of anesthesiologists, tracheal intubation and mechanical ventilation ensure a smooth and safe interventional treatment; during the operation, both a rigid bronchoscope and flexible endoscope are used to take advantage of their own particular advantages (145-148).

\section{Interventional treatment of peripheral lung cancer}

Most peripheral types of severe lung cancer are associated with old age, frailty, and poor lung function. Transbronchial biopsy and treatment techniques are being developed for early-stage peripheral lung cancer (149). Percutaneous interventional techniques are relatively more mature and have more clinical evidence of effectiveness and safety (150-152).

\section{Interventional treatment of hemoptysis in patients with lung cancer}

Hemoptysis, a common symptom of lung cancer, can occur before diagnosis and during treatment. Life-threatening 
hemoptysis may occur in a small proportion of lung cancer patients (153). Bronchial artery embolization is usually the preferred option for hemoptysis, with a success rate of 60-90\%. Transbronchial intervention techniques (including stenting) are also options (154).

\section{Interventional treatment of SVC syndrome}

The incidence of SVC syndrome is $2-4 \%$ in lung cancer patients and up to $10 \%$ in SCLC patients (155). When SVC syndrome causes laryngeal and cerebral edema and hemodynamic instability, urgent endovascular stenting is required (156).

\section{Consensus 9: application of anti-tumor drugs in patients with severe lung cancer}

Drug treatment is not contraindicated in all patients with severe lung cancer, and the key consideration is how to precisely use the selected drugs to achieve high efficiency with low toxicity. Currently, the medications for lung cancer mainly include chemotherapy, targeted therapy, antiangiogenic therapy, and immunotherapy. For targeted therapy, clinical studies have been carried out in patients with PS 0-3; for chemotherapy, antiangiogenic therapy, and immunotherapy, clinical studies have been conducted mainly in patients with PS 0 or 1 , with only a small amount of data from patients with PS 2 .

\section{Chemotherapy}

Clinical evidence for the use of platinum-based double agents in advanced NSCLC with a PS score of 2 (recommendation category: A; level of evidence: $1 \mathrm{a})$ In a meta-analysis of 12 randomized studies performed in NSCLC patients with PS 2 (4 dedicated to PS 2 and 8 stratified patients with PS 2), the platinum-based dual-agent combination group had a significantly higher survival benefit over the single-drug group $[\mathrm{HR}=0.71,95 \%$ confidence interval (CI): 0.61-0.81] (157). Another meta-analysis also confirmed that dual-agent chemotherapy significantly improved OS (HR $=0.72$; 95\% CI: 0.61-0.84; $\mathrm{P}<0.0001)$ and improved 1-year survival over single-drug chemotherapy, although the grade 3 to 4 hematologic toxicities also increased (158). An article summarizing 10 clinical studies reported a benefit of pemetrexed plus platinum in NSCLC patients with PS 2 over pemetrexed alone in both PFS $(\mathrm{HR}=0.46 ; \mathrm{P}<0.001)$ and $\mathrm{OS}(\mathrm{HR}=0.62 ; \mathrm{P}=0.001)(159)$.
A recent study showed that carboplatin combined with albumin-bound paclitaxel also had good efficacy (median PFS: 5.2 months; median OS: 14 months) (160). For advanced NSCLC patients with a PS of 2, platinumcontaining dual-drug chemotherapy is an option, but patient tolerability needs to be considered.

\section{Clinical evidence of chemotherapy for advanced NSCLC patients with a PS of 3 or 4 (recommendation} category: B; level of evidence: 2 b)

A phase II randomized controlled study comparing gemcitabine $\left(1,250 \mathrm{mg} / \mathrm{m}^{2}\right.$, days 1 and 8$) v s$. gemcitabine $\left(200 \mathrm{mg} / \mathrm{m}^{2}\right.$, day 1) plus cisplatin $\left(60 \mathrm{mg} / \mathrm{m}^{2}\right)$ in the treatment of advanced NSCLC with a PS of 2 or $3(75.9 \%$ had a PS score of 3 ) found that the low-dose combination chemotherapy was more effective than the single-agent chemotherapy (median PFS: 3.8 vs. 5.6 months; median OS: 4.3 vs. 6.8 months) and had fewer adverse effects (161). A retrospective study (66) included 96 patients with advanced NSCLC with PS $\geq 2$ who received chemotherapy, of whom $33.5 \%$ had a PS score of 3 or 4 . The most common chemotherapy regimen used was a combination of weekly paclitaxel $\left(60 \mathrm{mg} / \mathrm{m}^{2}\right)$ and carboplatin (AUC 2) in $57.8 \%$, followed by pemetrexed $500 \mathrm{mg} / \mathrm{m}^{2}$ plus carboplatin (AUC $5) /$ cisplatin every 3 weeks (16.8\%) and paclitaxel alone $(13.6 \%)$. Data analysis showed an overall response rate (ORR) of $20 \%$, a disease control rate (DCR) of $48.42 \%$, and a median PFS of 6.3 months; univariate analysis suggested that weekly paclitaxel plus carboplatin was associated with prolonged PFS, and $45.26 \%$ of the patients had an improved PS score during chemotherapy compared to baseline. However, there is little clinical evidence for chemotherapy application in NSCLC patients with a PS of 3 or 4 has been available. Most of these studies were retrospective studies, and there was a lack of prospective clinical research.

Clinical evidence of chemotherapy for advanced SCLC patients with a PS of 3 or 4 (recommendation category: A; level of evidence: 1a)

For extensive SCLC patients with a PS score of 3-4 caused by SCLC, the treatment recommended by the major guidelines is similar to that with a PS score of $0-2$. However, it is necessary to combine various factors and carefully choose the chemotherapy regimen, such as choosing a single drug or a reduced dose combination treatment. For poor PS scores caused by non-SCLC, the guidelines recommend the best supportive treatment. However, a retrospective 
study showed that chemotherapy significantly prolonged the survival of SCLC patients with a PS of 3 or 4 compared with best supportive treatment (162). There are also relatively few clinical studies on these patients.

\section{Targeted therapy}

Clinical evidence for EGFR-TKIs in advanced NSCLC patients with a $P S \geq 2$ (recommendation category: $A$; level of evidence: $1 \mathrm{~b}$ )

One study demonstrated good efficacy and good tolerability of gefitinib in treating advanced NSCLC patients with $E G F R$ mutation and $\mathrm{PS} \geq 2$ (163). A retrospective study performed in super-elderly NSCLC patients (aged $\geq 85$ years) with a PS score of 3-4 showed gefitinib had a greater OS benefit than best supportive care (4.6 vs. 2.3 months) (164). In a retrospective analysis, in patients with first or second generation TKI resistance and EGFR T790M mutationpositive NSCLC with PS scores of 2-4 who were treated with osimertinib, the ORR was 53\% (95\% CI: 36-70\%), the PS score improvement rate was $63 \%$, and the median PFS was 8.2 months. In addition, the treatment was well tolerated (165). Similarly, osimertinib also appears to be effective (median PFS: 7.0 months; median OS: 12.7 months) in a recent phase II trial enrolling 18 patients with T790Mmutated NSCLC with PS $\geq 2$, and it improved the PS score in $72 \%$ of the patients (166).

For EGFR-positive patients with a PS $\geq 2$, EGFR-TKI monotherapy remains the preferred regimen, and TKI combination therapy is currently less well documented.

\section{Clinical evidence of ALK-TKIs for advanced NSCLC patients with $\mathrm{PS} \geq \mathbf{2}$ (recommendation category: A; level of evidence: $1 b$ )}

A case report $(\mathrm{n}=5)$ suggested a promising efficacy for crizotinib in the treatment of advanced $A L K$ rearrangementpositive NSCLC patients with a PS $\geq 2$ (167). In another study, patients with advanced $A L K$ rearrangement-positive NSCLC and a PS of 2 to 4 received alectinib, which yielded an ORR of $72.2 \%$, a PFS of 10.1 months, and a PS improvement rate of $83.3 \%$ (168). The updated data showed that the median PFS reached 16.2 months, the median OS reached 30.3 months, and the 3 -year survival rate was $42 \%$ (169). A real-world study in Japan showed, after alectinib treatment, the OS was significantly shorter in patients with a PS score $\geq 2$ than those with a PS score $\leq 1$; however, the adverse reaction rates were similar, suggesting patients with poor PS scores could nonetheless tolerate alectinib (170).
Thus, ALK-TKIs may be a new option for $A L K$ rearrangement-positive NSCLC patients with a PS $\geq 2$, but is has only been explored in few clinical trials and deserves further verification in larger studies.

\section{Angiogenesis inhibitors (recommendation category: B; level of evidence: $1 b$ )}

A randomized phase II trial of pemetrexed, pemetrexed/ bevacizumab, and pemetrexed/carboplatin/bevacizumab in patients with advanced NSCLC and a PS of 2 showed that the PFS was $2.8,4.0$, and 4.8 months, respectively, and the ORR was $15 \%, 31 \%$, and $44 \%$, respectively, in these three groups (171). In a study with $92 \%$ of patients enrolled having PS of 2, the combination of erlotinib and bevacizumab also showed good efficacy and tolerability for recurrent NSCLC (172). A retrospective study showed that in the subgroup with a PS score of $\geq 2$, the HR for both PFS and OS was 0.47 in the bevacizumab combined with carboplatin + paclitaxel group compared to the chemotherapy alone group, although the difference was not statistically significant (173). Recently, one of our studies showed that, for patients with advanced squamous lung cancer with a PS score between 2 and 3 after failed secondor later-line systemic therapy, anlotinib plus S-1 prolonged survival compared with anlotinib alone (71).

Angiogenesis inhibitors have no specific PS score requirement and can be used as transitional options for patients with poor PS scores, and a new treatment regimen may be initiated after the PS score has improved.

\section{Immunotherapy (recommendation category: B; level of evidence: $1 b$ )}

The OS was shorter in PS 2 patients compared with PS 0 or 1 patients in CheckMate 153 (174) and CheckMate 171 (175), which included patients with advanced NSCLC treated with nivolumab, although the incidence of toxicities was comparable. In a clinical trial on NSCLC patients treated with pembrolizumab, the efficacy was similar between patients with NSCLC of PS 2 and those with PS 0 or 1 (regardless of PD-L1 expression), and no novel $\mathrm{AE}$ was observed (176). However, there were also studies performed in patients with PS 2 in which immunotherapy was ineffective $(177,178)$. Such data suggests that lung cancer patients with PS 2 are highly heterogeneous. It was found that patients with a PS of 2 as determined by comorbidities had significantly better outcomes compared 
with disease burden-induced PS 2 (179). Friedlaender et al. also proposed that, for SCLC patients with a poor PS score, the cause of the poor PS should be evaluated; immunotherapy might also be applied in SCLC patients with a PS of 2 after efficacy prediction (96). This is well in line with our treatment strategy. A case of a patient with a PS score of 3 and recurrent pulmonary infections due to $\mathrm{CAO}$. The patient received combination therapy including endoscopic recanalization of central airway followed by immunotherapy, which reduced tumor lesions and improved the PS score (180). Case reports have shown that immunotherapy was also effective in patients with a PS of $4(181,182)$. Nevertheless, the role of immunotherapy in patients with poor PS scores needs to be further investigated. Many studies of atezolizumab in treating elderly patients with advanced lung cancer have confirmed that the therapeutic benefits were similar between patients older than and younger than 65 years of age $(183,184)$; meanwhile, no novel AE was noted, and the side effects were basically predictable and manageable. Furthermore, many studies evaluating the efficacy of immunotherapy in patients with PS scores $\geq 2$ are in progress $(185,186)$, with an attempt to further clarify the survival benefits and clinical value of immunotherapy in patients with severe lung cancer.

\section{Consensus 10: application of life support techniques in patients with severe lung cancer}

Tumors originating in the lungs are often accompanied by other severe diseases. It is well known that the heart and lungs are the most important life-sustaining organs, and the failure of these two organs typically signals the end of life. While heart cancer is rare, lung cancer is one of the most common cancers worldwide. Therefore, life support including but not limited to the use of respiratory support techniques for patients with severe lung cancer is vital (recommendation category: B; level of evidence: $2 \mathrm{a}$ ).

\section{Appropriate respiratory support}

The appropriate respiratory support techniques should be selected based on the patients' respective conditions. Patients with severe lung cancer have a poor systemic status, and most of them also have poor lung compliance and are prone to hypoxemia or even respiratory failure. The main causes are diverse, mainly including: severe pneumonia, cardiogenic or non-cardiogenic pulmonary edema, lung injury caused by anti-tumor therapy (radiotherapy, targeted therapy, immunotherapy, etc.), aggravation of underlying diseases (e.g., COPD and ILD), and tumor complications (pulmonary embolism, airway obstruction, pleural effusion, pericardial effusion, etc.) (187). Respiratory failure may also occur postoperatively as a result of conditions such as atelectasis, pneumonia, pulmonary edema, and bronchopleural fistula (188).

At admission, patients with severe lung cancer should receive routine blood gas tests, assessment of respiratory function, and aggressive oxygen therapy with a nasal cannula or face mask to maintain an oxygen saturation level of 94-98\%. Based on the results of blood gas analysis and oxygenation monitoring, patients should be promptly judged to have respiratory failure (or not), and the timing of noninvasive and invasive ventilation should be carefully evaluated. The non-invasive ventilator ensures the quality of ventilation and can also set a balanced ratio of the spontaneous breaths according to the actual physical condition. It is better than the conventional oxygen inhalation in relieving dyspnea and can reduce the probability of intubation and the incidence of complications $(189,190)$.

\section{Supportive treatment of other vital organs}

Severe lung cancer can be associated with various types of organ dysfunction or even multi-organ failure. It is important to assess organ function in a timely manner, monitor intake/output, natriuretic peptide, cardiac enzyme profile, electrolytes and liver and kidney function, and perform electrocardiography and cardiac ultrasound in patients with severe lung cancer. Possible causes and triggers should be actively removed or mitigated and symptomatic supportive treatment of vital organs provided (e.g., heart, liver, kidney and brain) as soon as possible to prevent further deterioration. It is also necessary to correct electrolyte disorders and enhance fluid management to prevent hypovolemia or fluid overload. Supportive therapy with multiple devices (e.g., intra-aortic balloon counterpulsation, liver replacement therapy, renal replacement therapy, etc.) or tubes (nasogastric tube, urinary catheter, central venous catheter, etc.) may be considered in critically ill patients.

\section{Appropriate local therapy}

Malignant pleural effusion and pericardial effusion should be effectively drained. Tumor-induced tracheal obstruction can be relieved by interventional techniques. Body position can be adjusted so as to promote the drainage of respiratory 
secretions. Bedridden patients should be regularly turned and patted to avoid choking and mis-aspiration. Sputum can be aspirated at the bedside using a fiberoptic bronchoscope.

\section{Appropriate nutritional support therapy}

Nutritional support is essential to optimize the health status of lung cancer patients and maximize their ability to complete long-term cancer treatment. Studies have shown that $34.5-56.4 \%$ of lung cancer patients have malnutrition (191-193). Malnourished cancer patients have a reduced quality of life, a poorer prognosis, and a higher incidence of postoperative complications than well-nourished patients $(194,195)$.

Patients with severe lung cancer should be screened regularly for the risk for the presence of malnutrition. According to the ESPEN guidelines (196), patients should firstly be counseled on diet, and those who can eat should be encouraged to consume adequate energy (target energy intake: $25-30 \mathrm{cal} / \mathrm{kg}$; protein intake is recommended to be $1.0-1.5 \mathrm{~g} / \mathrm{kg} / \mathrm{d}$ ), and nutritional supplements can be added; for patients with feeding difficulties, enteral nutrition is preferred; and when nutritional needs cannot be met or enteral nutrition is contraindicated, parenteral nutrition can be selected. Supplementation of micronutrients can also be helpful, and an individualized nutrition regimen is recommended.

\section{Active anti-infective treatment}

Most lung cancer patients have chronic underlying diseases (e.g., COPD and bronchiectasis) and poor nutritional status and are affected by anti-tumor treatments (e.g., chemotherapy, radiotherapy, immunotherapy, and surgery), which lead to decreased immunity and/or damaged lung structure. As a result, these patients are more likely to suffer from respiratory tract infections $(197,198)$. In immunocompromised lung cancer patients, delayed anti-infective therapy increases the risks of secondary complications and infection-related death (199). Therefore, patients with severe lung cancer with co-existing infection should be given proper initial empirical antimicrobial therapy immediately. Specimens for culture must be collected prior to the administration of antimicrobials. Based on the clinical and epidemiological features, the antimicrobial regimen should be tailored to cover all of the likely pathogens. When the causative organism is not identified, broad-spectrum antibacterials (e.g., $\beta$-lactams/ enzyme inhibitors, third-generation cephalosporins/enzyme inhibitors, and carbapenems) are recommended. If there are obstructive cavity and poor drainage, which are more likely to be accompanied by anaerobic infection, the antibiotics selected should cover anaerobic bacteria; if routine antibiotic therapy fails, empirical antifungal therapy should be used as soon as possible, while also paying attention to the presence of any possible viral infection.

\section{Timely anticoagulation therapy}

Research has shown that patients with lung cancer combined with pulmonary embolism who do not undergo anticoagulation have a higher mortality rate than those treated with anticoagulants (78). The ASCO guidelines have recommended prophylactic anticoagulation in patients with malignancies who are at risk of VTE with a low risk of bleeding (200). Therefore, we recommend aggressive anticoagulation for patients with a confirmed VTE or risk of VTE. Based on the recommendations proposed by the International Society on Thrombosis and Haemostasis (ISTH) (201), the British Committee for the Standards in Haematology (BCSH) (202), and the ASCO (203), our anticoagulation strategies in lung cancer patients are as follows: for patients with cancer-related VTE with platelet counts above $50 \times 10^{9} / \mathrm{L}$, full-dose anticoagulation is recommended; for patients with platelet counts between $25 \times 10^{9} / \mathrm{L}$ and $50 \times 10^{9} / \mathrm{L}$, half-dose anticoagulation is acceptable; and for patients with platelet counts below $25 \times 10^{9} / \mathrm{L}$, anticoagulation is not recommended.

\section{Pulmonary rebabilitation}

In patients with lung cancer combined with COPD, preoperative pulmonary rehabilitation training can improve postoperative lung re-expansion and reduce postoperative complications (204-206). Postsurgical rehabilitation programs in patients surgically treated for lung cancer are also beneficial to improve the quality of life (207). Multidimensional exercise intervention can also improve physical and functional capacity, anxiety, and depression in patients with advanced-stage lung cancer $(208,209)$. Pulmonary rehabilitation includes exercise, education, nutritional support, and psychological support (210). Therefore, pulmonary rehabilitation requires multidisciplinary collaboration and joint efforts.

In conclusion, with the help of supportive care and antitumor treatment on the basis of dynamic clinical patients' assessment and precise molecular testing, patients with 
severe lung cancer are likely to achieve survival benefit, and improve PS score and their quality of life. Clinicians should pay close attention to severe lung cancer, search for its cause, and combine the results of precise detection and multidisciplinary opinions to provide individualized and comprehensive treatment for patients with severe lung cancer. We hope that this consensus will help in the treatment of severe lung cancer and encourage further research in this field.

\section{Questions to be further discussed and considered}

Do you think it is valuable for advanced lung cancer patients with PS score of 3-4 and gene driven negative to receive anti-cancer treatment?

\section{Expert opinion: Dr. Alfonso Fiorelli}

I believe that anti-cancer treatment may be valuable in selected patients with advanced lung cancer patients with PS score of 3-4 and gene driven negative. Due to better tolerance of immunotherapy compared with cytotoxic chemotherapy, it may lead to more liberal use in this subgroup of patients.

\section{Expert opinion: Dr. Francesco Petrella}

I think it would be a valuable option considering immunotherapy in stage IV younger patients without any immune system disease, although with PS score of 3-4. Combined chemo-radiotherapy-that should consider in stage IIIA/IIIB unresectable patients in good clinical conditionscould be questionable in PS 3-4 patients; in this group of patients, palliative radiotherapy and best support care could be taken into consideration, thus avoiding chemotherapy.

\section{Expert opinion: Dr. Hitoshi Igai}

No, I don't, because any treatment has the possibility of deteriorating the patient status except for radiation therapy or immunotherapy.

\section{Expert opinion: Dr. Nikolaos Tsoukalas}

In my opinion for this specific clinical scenario probably any anti-cancer treatment would not be valuable. Maybe we could think immunotherapy in patients with PS 3 and very high expression of PD-L1 or chemotherapy in patients with PS 3 and SCLC.

\section{Expert opinion: Dr. Piergiorgio Solli}

(I) Personally, I feel that any attempt should be done before giving up with anti-tumour therapies; in every case of lung cancer patient, even in PS 3-4. This should be seen as a continuous process, moving from assessing suitability for targeted or anti-angiogenic therapies and immunotherapy firstly then considering also conventional chemo, aiming to minimizing toxicity with low dose single-agent or combination regimen. Main goal should remain improvement in PS rather than prolonging survival or PFS.

(II) I am aware-as clearly stated in the manuscriptthat there is lack of literature evidence and studies are few and retrospective in nature. Still, due to the development of medical technology and to current knowledge, we nowadays experience that some of these patients can benefit for anti tumour conventional chemotherapy if it is adequately tailored to their specific clinical case.

Toxicity can be kept under control in most of the cases and the vast majority of co-morbidities can be counteracted and improved.

(III) There is a general restriction to this in my opinion, that are some of the severe cardiological associated disease (severe myocardial infarction with very depressed EF or important dilated cardiomyopathy).

(IV) These conditions might significantly impair the situation itself and have a higher impact on survival than lung cancer, often making impossible any chance of attempted cure.

\section{Expert opinion: Dr. Rossana Berardi}

I think that it could be valuable in selected cases of patients with PS score of 3-4.

\section{Expert opinion: Dr. Sara Bravaccini}

Yes (but only for specific cases).

\section{Expert opinion: Dr. Satoshi Watanabe}

Yes. SCLC patients with poor PS due to tumors should be treated with chemotherapy. Also, NSCLC patients with treatable complications, such as bacterial pneumonia and massive pleural effusion, should be treated.

\section{Expert opinion: Dr. Taichiro Goto}

This decision, of course, depends on the health condition of the patients, but I think PS score can be improved by the treatment in some cancer patients, especially if the patient's PS is closely associated with the tumor progression. In such recoverable PS cases, the treatment can be more aggressive 
than conventional ones.

\section{Expert opinion: Dr. Takeo Nakada}

This is a problem that requires careful judgment about the indications for treatment. If the decreased PS is clearly caused by the progression of the cancer, we consider aggressive treatment if the patients and their family fully understand and approve the increased likelihood of death associated with the treatment. Proper communication is essential to treat. However, I don't think it is indicated for the elderly or patients with complex comorbidities.

\section{Expert opinion: Dr. Yoshinobu Ichiki}

No. I think that anti-cancer treatment is generally not indicated for patients with advanced lung cancer of PS 3-4 who are negative for driver gene mutation. Since clinical trials of cytotoxic anticancer agents and ICIs are mainly conducted with good PS (PS 0-1), there is little evidence of poor PS cases, and the safety is unknown. I think that careful judgment is required so that drug therapy does not shorten the life span.

\section{Expert opinion: Dr. Yuichi Saito}

I think it is reasonable for physicians to consider the consensus for poor PS patients with not only advanced but also early-stage lung cancer.

\section{Expert opinion: Dr. Yuki Kataoka}

The answer from a medical point of view is almost "no". But in some cases, for example SCLC, and anecdotal episodes of ICIs, I sometimes provide treatment based on sufficient informed consent.

\section{Do you agree that there is a significant difference between severe lung cancer and end-stage lung cancer?}

\section{Expert opinion: Dr. Alfonso Fiorelli}

I fully agree. Generally, end-stage lung cancer is referred to patients who do not benefit of any specific treatments except palliative care to alleviate symptoms. A patient refers to the hospice may be an example of patient with end-stage of lung cancer. The goal of treatment in these subsets of patients is "to not prolong the survival but to improve the quality of survival" through palliative care. By contrast, patients with severe lung cancer should be referred to the hospital in order to undergo multimodality treatments. The goal is "to improve the clinical condition of these patients so that they become fit to receive additional treatments to prolong survival".

\section{Expert opinion: Dr. Francesco Petrella}

According to the description in the paper and to previous reports, I agree on the difference between "severe" and "advanced lung cancer". In particular, considering that the definition of "severe lung cancer" relies on performance status, we may define as severe lung cancer patients those presenting with early-stage lung cancer and impaired clinical conditions.

\section{Expert opinion: Dr. Hitoshi Igai}

Yes. I consider that severe lung cancer means the patient with severe comorbidities while end-stage lung cancer means that the patient is in the severe status due to the progression of the cancer and there is no effective treatment.

\section{Expert opinion: Dr. Nikolaos Tsoukalas}

Yes, I totally agree that these two clinical scenarios (severe lung cancer or end-stage lung cancer) are different. In particular, they have different treatment options (in severe lung cancer we can offer some anticancer treatments such as chemotherapy, targeted therapies, immunotherapy, radiotherapy or combinations but in end-stage lung cancer we should offer only best supportive care) and prognosis.

\section{Expert opinion: Dr. Piergiorgio Solli}

(I) I totally agree. It is a pioneer concept but truly linked with our routine daily practice. "End stage" -n my personal feeling-refers to an advanced disease that cannot be modified with any therapies. "Severe" refers rather to the setting (PS 3 or 4) that is determined not (not only) by the advanced stage of the lung cancer but to a miscellanea of conditions having an impact on performance status.

(II) This can be modified in the vast majority of patients and thus-while the first group (end stage) do not have any chance (few chances) of cure, the second (severe) can benefit from a combination of multidisciplinary, individualized and specific treatment including the full armamentarium of anti-tumour therapies (systemic, surgery, radiotherapy) and thus making a tailored treatment a viable option in an improved context (sort of combination of rehab + therapy).

(III) Moreover "severe" should be intended as a dynamic (not static) condition, because several lung cancer patients, regardless the initial stage of the disease, might fluctuate from PS $0-1$ to PS 2-4 and therefore 
became "simply" an early or advanced stage or a severe lung cancer case. How to deal with these cases, providing punctual and effective treatment, has become a very challenging real-world research topic.

\section{Expert opinion: Dr. Rossana Berardi} I do agree.

\section{Expert opinion: Dr. Sara Bravaccini}

Yes. I do agree.

\section{Expert opinion: Dr. Satoshi Watanabe}

Yes. I do agree.

\section{Expert opinion: Dr. Taichiro Goto}

I totally agree with the new concept of severe lung cancer, which is by definition different from end-stage lung cancer.

\section{Expert opinion: Dr. Takeo Nakada}

Yes. I do agree. Severe lung cancer and end-stage lung cancer should be clearly distinguished. I think that severe lung cancer is indicated for aggressive treatment, but endstage lung cancer is indicated for only palliative treatment. Palliative treatment may be required for both lung cancers. Palliative treatment includes symptomatology management focused on providing comfort and dignity.

\section{Expert opinion: Dr. Yoshinobu Ichiki}

Yes. I think there is a difference between severe lung cancer and end-stage lung cancer. The prognosis of severe lung cancer is determined by the disease status that lowers its PS score and the degree of lung cancer progression. If the disease status that lowers PS can be controlled, aggressive lung cancer treatment may be possible. On the other hand, end-stage lung cancer is always advanced, and although palliative care is possible, cancer treatment is considered difficult.

\section{Expert opinion: Dr. Yuichi Saito}

I finally understand what you want to advocate. However, it may be difficult to do it at first glance. So, I recommend you to explain the definition enough at the beginning of this manuscript.

\section{Expert opinion: Dr. Yuki Kataoka}

I believe that the situation is different between when the patient was diagnosed and when heavily treated.
With the development of medical technology at this stage, the best supportive treatment is not the only choice for patients with severe lung cancer. Do you agree that individualized anti-tumor treatment can prolong the survival of some patients with severe lung cancer

\section{Expert opinion: Dr. Alfonso Fiorelli}

I agree that individualized anti-tumor treatment can prolong the survival of some patients with severe lung cancer (see examples reported in question 5 reply).

\section{Expert opinion: Dr. Francesco Petrella}

I agree on this point; ideal candidates would be patients expressing gene-driven mutations or younger patients eligible to immunotherapy in advanced stages. Moreover, early-stage patients with impaired clinical conditions could be considered for limited resection or palliative radiotherapy on a case-by-case approach.

\section{Expert opinion: Dr. Hitoshi Igai}

Yes, I agree.

\section{Expert opinion: Dr. Nikolaos Tsoukalas}

Yes, I totally agree with this statement. Nowadays, of course we can offer some anti-cancer treatments in our patients with severe lung cancer apart from best supportive care. We should individualize the treatment plan for each patient with sever lung cancer and can offer some anti-cancer treatments such as chemotherapy, targeted therapies, immunotherapy, radiotherapy or combinations.

\section{Expert opinion: Dr. Piergiorgio Solli}

(I) I totally agree. Anti-cancer therapies + life support strategy + comorbidities management + dealing with complications should be seen as a synergic mechanism and do not conflict with each other. Many examples are available in clinical practice.

(II) Patient with SCLC might have life-threatening conditions (SVC syndrome, severe respiratory failure, others) and can benefit not only from the treatment of the critical disease but also from the cure of the oncological disorder. These two effects are mutual and empower the final result.

(III) The same applies to some of the haematological malignancies.

(IV) Best supportive therapy should be applied nowadays to a very minority of cases and only at the end of 
the pathway of lung cancer cases. At time of initial diagnosis there are many options, even for patients with compromised performance status.

\section{Expert opinion: Dr. Rossana Berardi}

I do agree, in selected patients.

\section{Expert opinion: Dr. Sara Bravaccini}

Yes. I do agree.

\section{Expert opinion: Dr. Satoshi Watanabe}

Yes. I do agree.

\section{Expert opinion: Dr. Taichiro Goto}

I agree with the authors. In this era of "Precision Medicine", diagnosis and treatment strategy to the lung cancer has been evolving dramatically in the field of circulating tumor DNA (ctDNA), liquid biopsy, gene sequencing, tumor mutation burden, genome profiling, and so on. Nowadays, individualized treatments can be devised and applied strategically and meticulously to the patients with severe lung cancer, while they are being validated in some ongoing studies.

\section{Expert opinion: Dr. Takeo Nakada}

I agree with this point. Certain treatments (such as TKIs) are expected to improve quality of life and prognosis in patients with positive driver mutations. Cytotoxic chemotherapy remains an integral part of cancer treatment. Clinicians should carefully select their patients.

\section{Expert opinion: Dr. Yoshinobu Ichiki}

Yes. I think that there are lung cancer patients who can be expected to prolong their survival time by individualized treatment even in patients with poor PS. Gefitinib for PS 3-4 EGFR mutation-positive patients and alectinib for $A L K$ fusion gene-positive patients have been reported to be effective against poor PS lung cancer patients, with 22 and 6 patients, respectively. However, no major safety issues were found [(I) Inoue et al., 7 Clin Oncol 2009, (65); (II) Iwama et al., 7 Thorac Oncol 2017, (168)].

\section{Expert opinion: Dr. Yuichi Saito}

Yes, I fully agree with it. In my opinion, best supportive treatment does not mean to reject all of treatments including surgery, chemotherapy, radiotherapy, etc. These aggressive treatments are often useful for improvement of quality of life.

\section{Expert opinion: Dr. Yuki Kataoka}

Yes. But we don't know how to individualize them now.

Do you agree that early-stage lung cancer also has severe state, but reasonable individualized treatment can also prolong the survival of patients?

\section{Expert opinion: Dr. Alfonso Fiorelli}

I agree that individualized treatments can prolong the survival in patients with early-stage lung cancer (see some examples reported in question 5 reply).

\section{Expert opinion: Dr. Francesco Petrella}

As described in the previous point, I think that severe, early-stage lung cancer patients-before being excluded form an effective therapeutic approach and considered for exclusive palliative treatment-should be evaluated for limited, parenchyma sparing procedures whenever feasible or SBRT in second instance.

\section{Expert opinion: Dr. Hitoshi Igai}

Yes, I agree.

\section{Expert opinion: Dr. Nikolaos Tsoukalas}

Yes, I absolutely agree with this comment for earlystage lung cancer that can be severe. Individualization of treatment-management oncology plan in patients with lung cancer is very important and can be crucial for their prognosis.

\section{Expert opinion: Dr. Piergiorgio Solli}

(I) Absolutely agree with this idea. I tried to explain this above. Severe lung cancer is a multifactorial concept and do not refer to lung cancer TNM stage.

(II) I personally believe that lung cancer management will soon look like breast cancer management where several options are available and personalized therapy will become the standard.

(III) Where with the term "personalized therapy" also optimal management of a wide range of comorbidities is included.

\section{Expert opinion: Dr. Rossana Berardi}

I do agree, in selected patients.

\section{Expert opinion: Dr. Sara Bravaccini}

Yes. I do agree. 


\section{Expert opinion: Dr. Satoshi Watanabe}

Yes. I do agree.

\section{Expert opinion: Dr. Taichiro Goto}

I agree with the authors, but those treatment, including SBRT, should be regarded as second-best way in terms of survival benefit. For the time being, surgery is the most appropriate therapy to early-stage lung cancers, and in case of inoperable patients, strict and reasonable indications should be applied to select the therapy most suitable for each patient.

\section{Expert opinion: Dr. Takeo Nakada}

Yes. I do agree.

\section{Expert opinion: Dr. Yoshinobu Ichiki}

Yes. I think that even in early-stage lung cancers with poor PS, the survival period can be expected to be extended by performing passive limited resection or radiation therapy after consulting with specialists.

\section{Expert opinion: Dr. Yuichi Saito}

Yes, I agree.

\section{Expert opinion: Dr. Yuki Kataoka}

I don't have enough experience and knowledge to answer this question.

Do you agree with the curative effect of precise radiotberapy and interventional technology in some patients with early severe lung cancer?

\section{Expert opinion: Dr. Alfonso Fiorelli}

Multimodality treatments including interventional treatments followed by radiotherapy, chemotherapy or immunotherapy, if indicated, may prolong survival of selected patients with early severe lung cancer. For example, a patient with malignant pleural effusion may benefit by invasive treatment of pleural effusion. This strategy improves the dyspnea and allows performing further therapy (standard chemotherapy, radiotherapy, immunotherapy, etc.) that can prolong survival. Additionally, patient with malignant obstruction of central airway and lung atelectasis is eligible for endoscopic recanalization of the central airway. This strategy allows releasing dyspnea so that he may undergo additional treatments (standard chemotherapy, radiotherapy, immunotherapy, etc.) aiming to prolong survival. I have recently published a case report on the beneficial effect of the combined treatment including endoscopic recanalization followed by immunotherapy in a patient who was previously not candidable to immunotherapy for the recurrent lung infection due to lobar atelectasis [Fiorelli et al., 7 Cardiothorac Surg 2019, (180)].

\section{Expert opinion: Dr. Francesco Petrella}

I agree on this point; ideal candidates would be $T_{1} / T_{2} N_{0}$ patients potentially eligible to SBRT or local ablations (e.g., thermo ablation, microwave ablation). Cardiopulmonary function, anyway, plays the pivotal role in this decisional process.

\section{Expert opinion: Dr. Hitoshi Igai}

Yes. Previous several reports described SBRT as effective for the frail patients with cStage I.

\section{Expert opinion: Dr. Nikolaos Tsoukalas}

Yes, I agree with the positive clinical impact of these treatment modalities (precise radiotherapy and interventional technology) not only in early severe lung cancer but also in oligometastatic disease.

\section{Expert opinion: Dr. Piergiorgio Solli}

Totally agree I tried to answer above. I am not a radiotherapist and I am not able to express more in details this.

\section{Expert opinion: Dr. Rossana Berardi}

Yes, I do.

\section{Expert opinion: Dr. Sara Bravaccini} Yes. I do agree.

\section{Expert opinion: Dr. Satoshi Watanabe}

Yes. I do agree.

\section{Expert opinion: Dr. Taichiro Goto}

I think, for example, curative effect of SBRT remains yet to be fully demonstrated as compared to surgery, thus both further development of technology and validation by largescale clinical trials are deemed necessary.

\section{Expert opinion: Dr. Takeo Nakada}

I agree radiotherapy and interventional technology for patients at high risk of surgery with early severe lung cancer. Patients who are tolerant for surgeries but strongly desire these treatments should be informed of the difficulty of surgery as an additional treatment after definitive radiation therapy and the predictive survival outcomes compared to surgery. 
Expert opinion: Dr. Yoshinobu Ichiki

Yes. Analysis of 53 cases of clinical stage I NSCLC, which could not tolerate lobectomy, showed that the 5-year survival rates for passive limited resection and definitive radiotherapy were $55.6 \%$ and $40.4 \%$, respectively, which tended to be favorable for passive limited surgery. However, there was no significant difference at $\mathrm{P}=0.124$ (119). At present, no clinical trials have been reported that randomly compare passive limited resection with definitive radiation therapy, but there are many reports showing acceptable results that may be considered.

\section{Expert opinion: Dr. Yuichi Saito}

In principle, surgical treatment should be considered even for patients with early severe lung cancer, however, I agree on that radiotherapy and/or interventional treatment could be better choice for such a poor PS patient.

\section{Expert opinion: Dr. Yuki Kataoka}

I want to know how you defined the "early severe lung cancer" to answer this question.

\section{Do you think chemotherapy and immunotherapy can be used in patients with severe lung cancer and prolong their survival?}

\section{Expert opinion: Dr. Alfonso Fiorelli}

I believe that in selected patients with severe lung cancer who are fit to receive chemotherapy and/or immunotherapy, these treatments can prolong survival. Obviously, before starting this strategy, it is mandatory to identify which patients can really have benefits from these treatments in the light of their high costs.

\section{Expert opinion: Dr. Francesco Petrella}

I think immunotherapy could be taken into consideration in younger metastatic patients suffering from severe lung cancer; on the contrary I do not believe that this cohort of patients may benefit from chemotherapy.

\section{Expert opinion: Dr. Hitoshi Igai}

Yes. Some drugs in chemotherapy or immunotherapy do not affect the patient's condition negatively.

\section{Expert opinion: Dr. Nikolaos Tsoukalas}

Yes absolutely! I have clinical experience of the positive clinical results that chemotherapy or immunotherapy can have in patients with severe lung cancer. The main issue is individualization of oncology treatment approach for each patient.

\section{Expert opinion: Dr. Piergiorgio Solli}

(I) Totally agree I tried to answer above. Immunotherapy for sure, due to a reduced toxicity and a better tolerability.

(II) The problem could be more complicated for standard chemotherapy due to lack of scientific evidence in this setting if lung cancer patients and due to a more challenging scenario.

\section{Expert opinion: Dr. Rossana Berardi}

I think that they can be used in selected patients.

\section{Expert opinion: Dr. Sara Bravaccini}

Yes. I think that they can be used in selected cases.

\section{Expert opinion: Dr. Satoshi Watanabe}

Yes. Systemic therapies could prolong survival in NSCLC patients with PS 2 and SCLC patients with PS 2-4 if the cause of poor PS is a lung cancer. If the cause of poor PS is a comorbidity, I do not think systemic therapies improve the prognosis of patients with lung cancers.

\section{Expert opinion: Dr. Taichiro Goto}

I would definitely say yes to this question. There are so many studies that demonstrated the efficacy of combined chemotherapy and immunotherapy. I believe novel combinations are also quite promising in the future.

\section{Expert opinion: Dr. Takeo Nakada}

I think chemotherapy and immunotherapy can prolong survival in some patients. Therefore, it is important to select patients who are indicated. I think it is necessary to build evidence and extract patients who can be expected to have therapeutic effects.

\section{Expert opinion: Dr. Yoshinobu Ichiki}

No. I think that anti-cancer treatment is generally not indicated for patients with advanced lung cancer of PS 3-4 who are negative for driver gene mutation. Since clinical trials of cytotoxic anticancer agents and ICIs are mainly conducted with good PS (PS 0-1), there is little evidence of poor PS cases, and the safety is unknown. I think that careful judgment is required so that drug therapy does not shorten the life span. Even with PS 3-4, molecular-targeted therapeutic agents may be considered in EGFR mutation- 
positive cases and $A L K$ fusion gene-positive cases.

\section{Expert opinion: Dr. Yuichi Saito}

Also, I agree on a case-by-case. Especially, pembrolizumab monotherapy could be better choice for patients with severe lung cancer when PD-L1 expression positive.

\section{Expert opinion: Dr. Yuki Kataoka}

The answer from a medical point of view is almost "no". But in some cases, for example SCLC, and anecdotal episodes of ICIs, I sometimes provide treatment based on sufficient informed consent.

\section{Acknowledgments}

The authors appreciate the academic support from AME Lung Cancer Collaborative Group.

Funding: None.

\section{Footnote}

Conflicts of Interest: All authors have completed the ICMJE uniform disclosure form (available at https://dx.doi. org/10.21037/tlcr-21-467). Y Song serves as an unpaid editorial board member of Translational Lung Cancer Research from Sep 2020 to Aug 2022. The other authors have no conflicts of interest to declare.

Ethical Statement: The authors are accountable for all aspects of the work in ensuring that questions related to the accuracy or integrity of any part of the work are appropriately investigated and resolved.

Open Access Statement: This is an Open Access article distributed in accordance with the Creative Commons Attribution-NonCommercial-NoDerivs 4.0 International License (CC BY-NC-ND 4.0), which permits the noncommercial replication and distribution of the article with the strict proviso that no changes or edits are made and the original work is properly cited (including links to both the formal publication through the relevant DOI and the license). See: https://creativecommons.org/licenses/by-nc-nd/4.0/.

\section{References}

1. Sung H, Ferlay J, Siegel RL, et al. Global Cancer Statistics 2020: GLOBOCAN Estimates of Incidence and Mortality Worldwide for 36 Cancers in 185 Countries. CA Cancer J
Clin 2021;71:209-49.

2. Maclay JD, Farley JM, McCowan C, et al. Obtaining tissue diagnosis in lung cancer patients with poor performance status and its influence on treatment and survival. Respir Med 2017;124:30-5.

3. Qin YY, Zhang DH, Lin XQ, et al. Clinical analysis of 36 cases of advanced non-small cell lung cancer (NSCLC) with performance status (PS) scores between 2 and 4 . Zhonghua Zhong Liu Za Zhi 2017;39:855-61.

4. Xie Z, Xu D, Jiang L, et al. Gefitinib in the treatment of 4 patients with severe advanced lung adenocarcinoma of PS 4: report of 4 cases and literature review. Medical Information 2014;(14):436-7.

5. Xie ZH, Zhou CZ, Qin YY, et al. Diagnosis and treatment strategy for advanced severe lung cancer. Chinese Journal of Practical Internal Medicine 2019;39:416-9.

6. Belot A, Fowler H, Njagi EN, et al. Association between age, deprivation and specific comorbid conditions and the receipt of major surgery in patients with non-small cell lung cancer in England: A population-based study. Thorax 2019;74:51-9.

7. Taioli E, Liu B, Nicastri DG, et al. Personal and hospital factors associated with limited surgical resection for lung cancer, in-hospital mortality and complications in New York State. J Surg Oncol 2017;116:471-81.

8. Group OLoEW. Oxford Centre for Evidence-Based Medicine 2011 Levels of Evidence. 2011. Available online: http://www.cebm.net/index.aspx?o=5653. Accessed 1 April 2021.

9. Grose D, Morrison DS, Devereux G, et al. Comorbidities in lung cancer: prevalence, severity and links with socioeconomic status and treatment. Postgrad Med J 2014;90:305-10.

10. Nieder C, Engljähringer K, Angelo K. Impact of comorbidity on survival after palliative radiotherapy. Strahlenther Onkol 2014;190:1149-53.

11. Colinet B, Jacot W, Bertrand D, et al. A new simplified comorbidity score as a prognostic factor in non-small-cell lung cancer patients: description and comparison with the Charlson's index. Br J Cancer 2005;93:1098-105.

12. Alexander M, Evans SM, Stirling RG, et al. The Influence of Comorbidity and the Simplified Comorbidity Score on Overall Survival in Non-Small Cell Lung Cancer-A Prospective Cohort Study. J Thorac Oncol 2016;11:748-57.

13. Grose D, Morrison DS, Devereux G, et al. The impact of comorbidity upon determinants of outcome in patients with lung cancer. Lung Cancer 2015;87:186-92. 
14. Ameri P, Canepa M, Anker MS, et al. Cancer diagnosis in patients with heart failure: epidemiology, clinical implications and gaps in knowledge. Eur J Heart Fail 2018;20:879-87.

15. de Boer RA, Meijers WC, van der Meer P, et al. Cancer and heart disease: associations and relations. Eur J Heart Fail 2019;21:1515-25.

16. Kravchenko J, Berry M, Arbeev K, et al. Cardiovascular comorbidities and survival of lung cancer patients: Medicare data based analysis. Lung Cancer 2015;88:85-93.

17. Loganathan RS, Stover DE, Shi W, et al. Prevalence of COPD in women compared to men around the time of diagnosis of primary lung cancer. Chest 2006;129:1305-12.

18. Buist AS, McBurnie MA, Vollmer WM, et al. International variation in the prevalence of COPD (the BOLD Study): a population-based prevalence study. Lancet 2007;370:741-50.

19. Lee SJ, Lee J, Park YS, et al. Impact of chronic obstructive pulmonary disease on the mortality of patients with nonsmall-cell lung cancer. J Thorac Oncol 2014;9:812-7.

20. Hashimoto N, Matsuzaki A, Okada Y, et al. Clinical impact of prevalence and severity of COPD on the decisionmaking process for therapeutic management of lung cancer patients. BMC Pulm Med 2014;14:14.

21. Lin H, Lu Y, Lin L, et al. Does chronic obstructive pulmonary disease relate to poor prognosis in patients with lung cancer?: A meta-analysis. Medicine (Baltimore) 2019;98:e14837.

22. Naccache JM, Gibiot Q, Monnet I, et al. Lung cancer and interstitial lung disease: a literature review. J Thorac Dis 2018;10:3829-44.

23. Tomassetti S, Gurioli C, Ryu JH, et al. The impact of lung cancer on survival of idiopathic pulmonary fibrosis. Chest 2015;147:157-64.

24. Raghu G, Amatto VC, Behr J, et al. Comorbidities in idiopathic pulmonary fibrosis patients: a systematic literature review. Eur Respir J 2015;46:1113-30.

25. Hyzy R, Huang S, Myers J, et al. Acute exacerbation of idiopathic pulmonary fibrosis. Chest 2007;132:1652-8.

26. Hoshikawa Y, Kondo T. Perioperative lung injury: acute exacerbation of idiopathic pulmonary fibrosis and acute interstitial pneumonia after pulmonary resection. Nihon Geka Gakkai Zasshi 2004;105:757-62.

27. Kumar P, Goldstraw P, Yamada K, et al. Pulmonary fibrosis and lung cancer: risk and benefit analysis of pulmonary resection. J Thorac Cardiovasc Surg 2003;125:1321-7.

28. Miyamoto A, Kishi K, Yoshimura K. A nationwide survey concerning lung surgery for lung cancer associated with idiopathic interstitial pneumonia. Nihon Kokyuki Gakkai Zasshi 2011;49:148-50.

29. Saito Y, Kawai Y, Takahashi N, et al. Survival after surgery for pathologic stage IA non-small cell lung cancer associated with idiopathic pulmonary fibrosis. Ann Thorac Surg 2011;92:1812-7.

30. Hidayat K, Du X, Chen G, et al. Abdominal Obesity and Lung Cancer Risk: Systematic Review and Meta-Analysis of Prospective Studies. Nutrients 2016;8:810.

31. Salome CM, King GG, Berend N. Physiology of obesity and effects on lung function. J Appl Physiol (1985) 2010;108:206-11.

32. Zerah F, Harf A, Perlemuter L, et al. Effects of obesity on respiratory resistance. Chest 1993;103:1470-6.

33. Bamgbade OA, Rutter TW, Nafiu OO, et al. Postoperative complications in obese and nonobese patients. World J Surg 2007;31:556-60; discussion 561.

34. De Jong A, Verzilli D, Chanques G, et al. Preoperative risk and perioperative management of obese patients. Rev Mal Respir 2019;36:985-1001.

35. Gompelmann D, Eberhardt R, Herth FJ. Advanced malignant lung disease: what the specialist can offer. Respiration 2011;82:111-23.

36. Porcel JM, Gasol A, Bielsa S, et al. Clinical features and survival of lung cancer patients with pleural effusions. Respirology 2015;20:654-9.

37. Porcel JM. Malignant pleural effusions because of lung cancer. Curr Opin Pulm Med 2016;22:356-61.

38. Ryu JS, Ryu HJ, Lee SN, et al. Prognostic impact of minimal pleural effusion in non-small-cell lung cancer. J Clin Oncol 2014;32:960-7.

39. Yoneda KY, Mathur PN, Gasparini S. The evolving role of interventional pulmonary in the interdisciplinary approach to the staging and management of lung cancer. Part III: diagnosis and management of malignant pleural effusions. Clin Lung Cancer 2007;8:535-47.

40. Ou SH, Zell JA. Validation study of the proposed IASLC staging revisions of the T4 and M non-small cell lung cancer descriptors using data from 23,583 patients in the California Cancer Registry. J Thorac Oncol 2008;3:216-27.

41. Tsang TS, Seward JB, Barnes ME, et al. Outcomes of primary and secondary treatment of pericardial effusion in patients with malignancy. Mayo Clin Proc 2000;75:248-53.

42. Gornik HL, Gerhard-Herman M, Beckman JA. Abnormal cytology predicts poor prognosis in cancer patients with pericardial effusion. J Clin Oncol 2005;23:5211-6.

43. Rousseau-Bussac G, Crequit P, Alifano M, et al. 
Management of malignant pericardial effusion in lung cancer. Rev Mal Respir 2014;31:746-53.

44. Hu ZG, Hu K, Li WX, et al. Prognostic factors and nomogram for cancer-specific death in non small cell lung cancer with malignant pericardial effusion. PLoS One 2019; 14:e0217007.

45. Di Liso E, Menichetti A, Dieci MV, et al. Neoplastic Pericardial Effusion: A Monocentric Retrospective Study. J Palliat Med 2019;22:691-5.

46. Guibert N, Mazieres J, Marquette CH, et al. Integration of interventional bronchoscopy in the management of lung cancer. Eur Respir Rev 2015;24:378-91.

47. Chen Y, Zhou Z, Feng J, et al. Use of Hybrid Stent in Management of Complex Malignant Airway Stenosisand Fistulas. Zhongguo Fei Ai Za Zhi 2020;23:472-8.

48. Skowronek J, Młynarczyk W, Piorunek T. Brachytherapy in the treatment of lung and tracheal cancer: current indications, methods, results. Przegl Lek 2006;63:664-73.

49. Connolly GC, Dalal M, Lin J, et al. Incidence and predictors of venous thromboembolism (VTE) among ambulatory patients with lung cancer. Lung Cancer 2012;78:253-8.

50. Walker AJ, Card TR, West J, et al. Incidence of venous thromboembolism in patients with cancer - a cohort study using linked United Kingdom databases. Eur J Cancer 2013;49:1404-13.

51. Khorana AA, Francis CW, Culakova E, et al. Thromboembolism is a leading cause of death in cancer patients receiving outpatient chemotherapy. J Thromb Haemost 2007;5:632-4.

52. Serpa Neto A, Hemmes SN, Barbas CS, et al. Incidence of mortality and morbidity related to postoperative lung injury in patients who have undergone abdominal or thoracic surgery: a systematic review and meta-analysis. Lancet Respir Med 2014;2:1007-15.

53. Ali FS, Arevalo O, Zorofchian S, et al. Cerebral Radiation Necrosis: Incidence, Pathogenesis, Diagnostic Challenges, and Future Opportunities. Curr Oncol Rep 2019;21:66.

54. Rahmathulla G, Marko NF, Weil RJ. Cerebral radiation necrosis: a review of the pathobiology, diagnosis and management considerations. J Clin Neurosci 2013;20:485-502.

55. Li Y, Klippel Z, Shih X, et al. Relationship between severity and duration of chemotherapy-induced neutropenia and risk of infection among patients with nonmyeloid malignancies. Support Care Cancer 2016;24:4377-83.

56. Cupp J, Culakova E, Poniewierski MS, et al. Analysis of
Factors Associated With In-hospital Mortality in Lung Cancer Chemotherapy Patients With Neutropenia. Clin Lung Cancer 2018;19:e163-9.

57. Hapani S, Sher A, Chu D, et al. Increased risk of serious hemorrhage with bevacizumab in cancer patients: a metaanalysis. Oncology 2010;79:27-38.

58. Qi WX, Sun YJ, Shen Z, et al. Risk of interstitial lung disease associated with EGFR-TKIs in advanced nonsmall-cell lung cancer: a meta-analysis of 24 phase III clinical trials. J Chemother 2015;27:40-51.

59. Suh CH, Kim KW, Pyo J, et al. The incidence of ALK inhibitor-related pneumonitis in advanced non-smallcell lung cancer patients: A systematic review and metaanalysis. Lung Cancer 2019;132:79-86.

60. Suresh K, Naidoo J, Lin CT, et al. Immune Checkpoint Immunotherapy for Non-Small Cell Lung Cancer: Benefits and Pulmonary Toxicities. Chest 2018;154:1416-23.

61. Suresh K, Voong KR, Shankar B, et al. Pneumonitis in Non-Small Cell Lung Cancer Patients Receiving Immune Checkpoint Immunotherapy: Incidence and Risk Factors. J Thorac Oncol 2018;13:1930-9.

62. Cho JY, Kim J, Lee JS, et al. Characteristics, incidence, and risk factors of immune checkpoint inhibitor-related pneumonitis in patients with non-small cell lung cancer. Lung Cancer 2018;125:150-6.

63. Tone M, Izumo T, Awano N, et al. High mortality and poor treatment efficacy of immune checkpoint inhibitors in patients with severe grade checkpoint inhibitor pneumonitis in non-small cell lung cancer. Thorac Cancer 2019;10:2006-12.

64. Lin X, Deng H, Chen L, et al. Clinical types of checkpoint inhibitor-related pneumonitis in lung cancer patients: a multicenter experience. Transl Lung Cancer Res 2021;10:415-29.

65. Inoue A, Kobayashi K, Usui K, et al. First-line gefitinib for patients with advanced non-small-cell lung cancer harboring epidermal growth factor receptor mutations without indication for chemotherapy. J Clin Oncol 2009;27:1394-400.

66. Kancharla H, Gundu N, Pathak N, et al. Cytotoxic chemotherapy in advanced non-small cell lung cancer with poor performance status: A retrospective analysis from routine clinical practice. Curr Probl Cancer 2020;44:100550.

67. Celermajer DS, Boyer MJ, Bailey BP, et al. Pericardiocentesis for symptomatic malignant pericardial effusion: a study of 36 patients. Med J Aust 
1991;154:19-22.

68. Martinoni A, Cipolla CM, Civelli M, et al. Intrapericardial treatment of neoplastic pericardial effusions. Herz 2000;25:787-93.

69. Lewis MA, Hendrickson AW, Moynihan TJ. Oncologic emergencies: Pathophysiology, presentation, diagnosis, and treatment. CA Cancer J Clin 2011;61:287-314.

70. Grose D, Devereux G, Brown L, et al. Variation in comorbidity and clinical management in patients newly diagnosed with lung cancer in four Scottish centers. J Thorac Oncol 2011;6:500-9.

71. Xie XH, Wang F, Lin XQ, et al. Anlotinib Plus S-1 for Patients with EGFR Mutation-Negative Advanced Squamous Cell Lung Cancer with PS Scores of 2-3 After Progression of Second-Line or Later-Line Treatment. Cancer Manag Res 2020;12:12709-14.

72. Keats JJ, Chesi M, Egan JB, et al. Clonal competition with alternating dominance in multiple myeloma. Blood 2012;120:1067-76.

73. Almendro V, Marusyk A, Polyak K. Cellular heterogeneity and molecular evolution in cancer. Annu Rev Pathol 2013;8:277-302.

74. Santarpia M, Liguori A, D'Aveni A, et al. Liquid biopsy for lung cancer early detection. J Thorac Dis 2018;10:S882-97.

75. Russo A, De Miguel Perez D, Gunasekaran M, et al. Liquid biopsy tracking of lung tumor evolutions over time. Expert Rev Mol Diagn 2019;19:1099-108.

76. Zhang J, Zhou JB, Lin XF, et al. Prevalence of undiagnosed and undertreated chronic obstructive pulmonary disease in lung cancer population. Respirology 2013;18:297-302.

77. Wang F, Xie XH, Lin XQ, et al. Exploration of the treatment model for patients with advanced non-small cell lung cancer complicated with chronic obstructive pulmonary disease based on real-world data. Zhonghua Jie He He Hu Xi Za Zhi 2020;43:450-4.

78. Sun JM, Kim TS, Lee J, et al. Unsuspected pulmonary emboli in lung cancer patients: the impact on survival and the significance of anticoagulation therapy. Lung Cancer 2010;69:330-6.

79. Sève $P$, Dumontet C. Chemoresistance in non-small cell lung cancer. Curr Med Chem Anticancer Agents 2005;5:73-88.

80. Bepler G, Kusmartseva I, Sharma S, et al. RRM1 modulated in vitro and in vivo efficacy of gemcitabine and platinum in non-small-cell lung cancer. J Clin Oncol 2006;24:4731-7.

81. Yang JC, Cheng Y, Murakami H, et al. A Randomized
Phase 2 Study of Gefitinib With or Without Pemetrexed as First-line Treatment in Nonsquamous NSCLC With EGFR Mutation: Final Overall Survival and Biomarker Analysis. J Thorac Oncol 2020;15:91-100.

82. Azuma K, Sasada T, Kawahara A, et al. Expression of ERCC1 and class III beta-tubulin in non-small cell lung cancer patients treated with carboplatin and paclitaxel. Lung Cancer 2009;64:326-33.

83. Westover D, Zugazagoitia J, Cho BC, et al. Mechanisms of acquired resistance to first- and second-generation EGFR tyrosine kinase inhibitors. Ann Oncol 2018;29:i10-9.

84. Qiang H, Chang Q, Xu J, et al. New advances in antiangiogenic combination therapeutic strategies for advanced non-small cell lung cancer. J Cancer Res Clin Oncol 2020;146:631-45.

85. Dowlati A, Gray R, Sandler AB, et al. Cell adhesion molecules, vascular endothelial growth factor, and basic fibroblast growth factor in patients with non-small cell lung cancer treated with chemotherapy with or without bevacizumab--an Eastern Cooperative Oncology Group Study. Clin Cancer Res 2008;14:1407-12.

86. Mok T, Gorbunova V, Juhasz E, et al. A correlative biomarker analysis of the combination of bevacizumab and carboplatin-based chemotherapy for advanced nonsquamous non-small-cell lung cancer: results of the phase II randomized ABIGAIL study (BO21015). J Thorac Oncol 2014;9:848-55.

87. Aguiar PN Jr, De Mello RA, Hall P, et al. PD-L1 expression as a predictive biomarker in advanced non-small-cell lung cancer: updated survival data. Immunotherapy 2017;9:499-506.

88. Le DT, Uram JN, Wang H, et al. PD-1 Blockade in Tumors with Mismatch-Repair Deficiency. N Engl J Med 2015;372:2509-20.

89. Lemery S, Keegan P, Pazdur R. First FDA Approval Agnostic of Cancer Site - When a Biomarker Defines the Indication. N Engl J Med 2017;377:1409-12.

90. Hellmann MD, Ciuleanu TE, Pluzanski A, et al. Nivolumab plus Ipilimumab in Lung Cancer with a High Tumor Mutational Burden. N Engl J Med 2018;378:2093-104.

91. Wang F, Zhao Q, Wang YN, et al. Evaluation of POLE and POLD1 Mutations as Biomarkers for Immunotherapy Outcomes Across Multiple Cancer Types. JAMA Oncol 2019;5:1504-6.

92. Walk EE, Yohe SL, Beckman A, et al. The Cancer Immunotherapy Biomarker Testing Landscape. Arch Pathol Lab Med 2020;144:706-24. 
93. Kato S, Goodman A, Walavalkar V, et al. Hyperprogressors after Immunotherapy: Analysis of Genomic Alterations Associated with Accelerated Growth Rate. Clin Cancer Res 2017;23:4242-50.

94. Forschner A, Hilke FJ, Bonzheim I, et al. MDM2, MDM4 and EGFR Amplifications and Hyperprogression in Metastatic Acral and Mucosal Melanoma. Cancers (Basel) 2020;12:540.

95. Chen Y, Huang Y, Gao X, et al. CCND1 Amplification Contributes to Immunosuppression and Is Associated With a Poor Prognosis to Immune Checkpoint Inhibitors in Solid Tumors. Front Immunol 2020;11:1620.

96. Friedlaender A, Liu SV, Passaro A, et al. The Role of Performance Status in Small-Cell Lung Cancer in the Era of Immune Checkpoint Inhibitors. Clin Lung Cancer 2020;21:e539-43.

97. Socinski MA, Jotte RM, Cappuzzo F, et al. Atezolizumab for First-Line Treatment of Metastatic Nonsquamous NSCLC. N Engl J Med 2018;378:2288-301.

98. Saito H, Fukuhara T, Furuya N, et al. Erlotinib plus bevacizumab versus erlotinib alone in patients with EGFR-positive advanced non-squamous non-small-cell lung cancer (NEJ026): interim analysis of an open-label, randomised, multicentre, phase 3 trial. Lancet Oncol 2019;20:625-35.

99. Bossert J, Forstner J, Villalobos M, et al. What patients with lung cancer with comorbidity tell us about interprofessional collaborative care across healthcare sectors: qualitative interview study. BMJ Open 2020;10:e036495.

100. Harada H, Yamashita Y, Misumi K, et al. Multidisciplinary team-based approach for comprehensive preoperative pulmonary rehabilitation including intensive nutritional support for lung cancer patients. PLoS One 2013;8:e59566.

101.Ellis PM. The importance of multidisciplinary team management of patients with non-small-cell lung cancer. Curr Oncol 2012;19:S7-S15.

102. Bilfinger TV, Albano D, Perwaiz M, et al. Survival Outcomes Among Lung Cancer Patients Treated Using a Multidisciplinary Team Approach. Clin Lung Cancer 2018;19:346-51.

103. Kowalczyk A, Jassem J. Multidisciplinary team care in advanced lung cancer. Transl Lung Cancer Res 2020;9:1690-8.

104. Pahlkotter MK, Mohidul S, Moen MR, et al. BMI and VTE Risk in Emergency General Surgery, Does Size Matter? : An ACS-NSQIP Database Analysis. Am Surg
2020;86:1660-5.

105.Ji X, Ding H. The efficacy of enteral nutrition combined with accelerated rehabilitation in non-small cell lung cancer surgery: A randomized controlled trial protocol. Medicine (Baltimore) 2020;99:e23382.

106.ATS Committee on Proficiency Standards for Clinical Pulmonary Function Laboratories. ATS statement: guidelines for the six-minute walk test. Am J Respir Crit Care Med 2002;166:111-7.

107.Agnew N. Preoperative cardiopulmonary exercise testing. Continuing Education in Anaesthesia Critical Care \& Pain 2010;10:33-7.

108. Wang J. The research of predicting the postoperative complications in patients with lung cancer using cardiopulmonary exercise test and 6-minute walk test. Master's dissertation. Tianjin: Tianjin Medical University, 2013.

109. Sardari Nia P, Olsthoorn JR, Heuts S, et al. Interactive 3D Reconstruction of Pulmonary Anatomy for Preoperative Planning, Virtual Simulation, and Intraoperative Guiding in Video-Assisted Thoracoscopic Lung Surgery. Innovations (Phila) 2019;14:17-26.

110. Ginsberg RJ, Rubinstein LV. Randomized trial of lobectomy versus limited resection for T1 N0 non-small cell lung cancer. Lung Cancer Study Group. Ann Thorac Surg 1995;60:615-22; discussion 622-3.

111. Yacoub WN, Meyers BF. Surgical resection in combination with lung volume reduction surgery for stage I nonsmall cell lung cancer. Semin Thorac Cardiovasc Surg 2010;22:38-43.

112. Choong CK, Meyers BF, Battafarano RJ, et al. Lung cancer resection combined with lung volume reduction in patients with severe emphysema. J Thorac Cardiovasc Surg 2004;127:1323-31.

113. Kushibe K, Takahama M, Tojo T, et al. Assessment of pulmonary function after lobectomy for lung cancer-upper lobectomy might have the same effect as lung volume reduction surgery. Eur J Cardiothorac Surg 2006;29:886-90.

114.Zhang $W$, Chen $M$, Li H, et al. Hypoxia preconditioning attenuates lung injury after thoracoscopic lobectomy in patients with lung cancer: a prospective randomized controlled trial. BMC Anesthesiol 2019;19:209.

115. Nannini LJ, Poole P, Milan SJ, et al. Combined corticosteroid and long-acting beta(2)-agonist in one inhaler versus inhaled corticosteroids alone for chronic obstructive pulmonary disease. Cochrane Database Syst Rev 2013;2013:CD006826. 
116. Gautam SS, O'Toole RF. Convergence in the Epidemiology and Pathogenesis of COPD and Pneumonia. COPD 2016;13:790-8.

117. Cui F, Liu J, Shao W, et al. Thoracoscopic minimally invasive surgery for non-small cell lung cancer in patients with chronic obstructive pulmonary disease. J Thorac Dis 2013;5 Suppl 3:S260-6.

118. Hoshikawa Y, Tochii D. Postoperative Atelectasis and Pneumonia after General Thoracic Surgery. Kyobu Geka 2017;70:649-55.

119. Matsuo Y, Chen F, Hamaji M, et al. Comparison of long-term survival outcomes between stereotactic body radiotherapy and sublobar resection for stage I non-smallcell lung cancer in patients at high risk for lobectomy: A propensity score matching analysis. Eur J Cancer 2014;50:2932-8.

120. Videtic GMM, Donington J, Giuliani M, et al. Stereotactic body radiation therapy for early-stage non-small cell lung cancer: Executive Summary of an ASTRO Evidence-Based Guideline. Pract Radiat Oncol 2017;7:295-301.

121. Guckenberger M, Andratschke N, Dieckmann K, et al. ESTRO ACROP consensus guideline on implementation and practice of stereotactic body radiotherapy for peripherally located early stage non-small cell lung cancer. Radiother Oncol 2017;124:11-7.

122. Tandberg DJ, Tong BC, Ackerson BG, et al. Surgery versus stereotactic body radiation therapy for stage I nonsmall cell lung cancer: A comprehensive review. Cancer 2018;124:667-78.

123. Verma V, Simone CB 2nd, Allen PK, et al. MultiInstitutional Experience of Stereotactic Ablative Radiation Therapy for Stage I Small Cell Lung Cancer. Int J Radiat Oncol Biol Phys 2017;97:362-71.

124. Shioyama Y, Onishi H, Takayama K, et al. Clinical Outcomes of Stereotactic Body Radiotherapy for Patients with Stage I Small-Cell Lung Cancer: Analysis of a Subset of the Japanese Radiological Society Multi-Institutional SBRT Study Group Database. Technol Cancer Res Treat 2018;17:1533033818783904.

125. Yang CJ, Chan DY, Shah SA, et al. Long-term Survival After Surgery Compared With Concurrent Chemoradiation for Node-negative Small Cell Lung Cancer. Ann Surg 2018;268:1105-12.

126. Aupérin A, Le Péchoux C, Rolland E, et al. Meta-analysis of concomitant versus sequential radiochemotherapy in locally advanced non-small-cell lung cancer. J Clin Oncol 2010;28:2181-90.

127. Curran WJ Jr, Paulus R, Langer CJ, et al. Sequential vs. concurrent chemoradiation for stage III non-small cell lung cancer: randomized phase III trial RTOG 9410. J Natl Cancer Inst 2011;103:1452-60.

128. Bi N, Wang L, Xu K, et al. Real-world treatment patterns and clinical outcomes in EGFR-mutant unresectable locally advanced NSCLC (LA-NSCLC): A retrospective multicenter study of 367 patients. Journal of Clinical Oncology 2020;38:abstr 9047.

129. Yamamoto M, Serizawa T, Shuto T, et al. Stereotactic radiosurgery for patients with multiple brain metastases (JLGK0901): a multi-institutional prospective observational study. Lancet Oncol 2014;15:387-95.

130. Chang EL, Wefel JS, Hess KR, et al. Neurocognition in patients with brain metastases treated with radiosurgery or radiosurgery plus whole-brain irradiation: a randomised controlled trial. Lancet Oncol 2009;10:1037-44.

131. Ernani V, Stinchcombe TE. Management of Brain Metastases in Non-Small-Cell Lung Cancer. J Oncol Pract 2019;15:563-70.

132. Bi N, Ma Y, Xiao J, et al. A Phase II Trial of Concurrent Temozolomide and Hypofractionated Stereotactic Radiotherapy for Complex Brain Metastases. Oncologist 2019;24:e914-20.

133. Sun $\mathrm{H}, \mathrm{Xu} \mathrm{L}$, Wang Y, et al. Additional radiation boost to whole brain radiation therapy may improve the survival of patients with brain metastases in small cell lung cancer. Radiat Oncol 2018;13:250.

134. Ni M, Jiang A, Liu W, et al. Whole brain radiation therapy plus focal boost may be a suitable strategy for brain metastases in SCLC patients: a multi-center study. Radiat Oncol 2020;15:70.

135.Li J, He J, Cai L, et al. Bevacizumab as a treatment for radiation necrosis following stereotactic radiosurgery for brain metastases: clinical and radiation dosimetric impacts. Ann Palliat Med 2021;10:2018-26.

136.Zhen J, Wen L, Lai M, et al. Whole brain radiotherapy (WBRT) for leptomeningeal metastasis from NSCLC in the era of targeted therapy: a retrospective study. Radiat Oncol 2020;15:185.

137.Su SF, Hu YX, Ouyang WW, et al. Overall survival and toxicities regarding thoracic three-dimensional radiotherapy with concurrent chemotherapy for stage IV non-small cell lung cancer: results of a prospective singlecenter study. BMC Cancer 2013;13:474.

138. Moeller B, Balagamwala EH, Chen A, et al. Palliative thoracic radiation therapy for non-small cell lung cancer: 2018 Update of an American Society for Radiation Oncology (ASTRO) Evidence-Based Guideline. Pract 
Radiat Oncol 2018;8:245-50.

139.Petrelli F, Ghidini A, Cabiddu M, et al. Addition of radiotherapy to the primary tumour in oligometastatic NSCLC: A systematic review and meta-analysis. Lung Cancer 2018;126:194-200.

140.Su S, Li T, Lu B, et al. Three-Dimensional Radiation Therapy to the Primary Tumor with Concurrent Chemotherapy in Patients With Stage IV Non-Small Cell Lung Cancer: Results of a Multicenter Phase 2 Study From PPRA-RTOG, China. Int J Radiat Oncol Biol Phys 2015;93:769-77.

141. Gomez DR, Tang C, Zhang J, et al. Local Consolidative Therapy Vs. Maintenance Therapy or Observation for Patients with Oligometastatic Non-Small-Cell Lung Cancer: Long-Term Results of a Multi-Institutional, Phase II, Randomized Study. J Clin Oncol 2019;37:1558-65.

142. Gomez DR, Blumenschein GR Jr, Lee JJ, et al. Local consolidative therapy versus maintenance therapy or observation for patients with oligometastatic non-smallcell lung cancer without progression after first-line systemic therapy: a multicentre, randomised, controlled, phase 2 study. Lancet Oncol 2016;17:1672-82.

143. Wahidi MM, Herth FJF, Chen A, et al. State of the Art: Interventional Pulmonology. Chest 2020;157:724-36.

144.Kalsi HS, Thakrar R, Gosling AF, et al. Interventional Pulmonology: A Brave New World. Thorac Surg Clin 2020;30:321-38.

145. Ost DE, Ernst A, Grosu HB, et al. Therapeutic bronchoscopy for malignant central airway obstruction: success rates and impact on dyspnea and quality of life. Chest 2015;147:1282-98.

146. Ong P, Grosu HB, Debiane L, et al. Long-term qualityadjusted survival following therapeutic bronchoscopy for malignant central airway obstruction. Thorax 2019;74:141-56.

147. Ost DE, Ernst A, Grosu HB, et al. Complications Following Therapeutic Bronchoscopy for Malignant Central Airway Obstruction: Results of the AQuIRE Registry. Chest 2015;148:450-71.

148.Jin F, Li Q, Li S, et al. Interventional Bronchoscopy for the Treatment of Malignant Central Airway Stenosis: An Expert Recommendation for China. Respiration 2019;97:484-94.

149. Chaddha U, Kurman JS, Mahajan A, et al. Lung Nodule Management: An Interventional Pulmonology Perspective. Semin Respir Crit Care Med 2018;39:661-6.

150.Smith SL, Jennings PE. Lung radiofrequency and microwave ablation: a review of indications, techniques and post-procedural imaging appearances. Br J Radiol 2015;88:20140598.

151.Li G, Xue M, Chen W, et al. Efficacy and safety of radiofrequency ablation for lung cancers: A systematic review and meta-analysis. Eur J Radiol 2018;100:92-8.

152. Tafti BA, Genshaft S, Suh R, et al. Lung Ablation: Indications and Techniques. Semin Intervent Radiol 2019;36:163-75.

153. Kathuria H, Hollingsworth HM, Vilvendhan R, et al. Management of life-threatening hemoptysis. J Intensive Care 2020;8:23.

154. Gershman E, Guthrie R, Swiatek K, et al. Management of hemoptysis in patients with lung cancer. Ann Transl Med 2019;7:358.

155.Azizi AH, Shafi I, Shah N, et al. Superior Vena Cava Syndrome. JACC Cardiovasc Interv 2020;13:2896-910.

156. Lanciego C, Pangua C, Chacón JI, et al. Endovascular stenting as the first step in the overall management of malignant superior vena cava syndrome. AJR Am J Roentgenol 2009;193:549-58.

157.Mörth C, Valachis A. Single-agent versus combination chemotherapy as first-line treatment for patients with advanced non-small cell lung cancer and performance status 2: a literature-based meta-analysis of randomized studies. Lung Cancer 2014;84:209-14.

158. Luo L, Hu Q, Jiang JX, et al. Comparing single-agent with doublet chemotherapy in first-line treatment of advanced non-small cell lung cancer with performance status 2: a meta-analysis. Asia Pac J Clin Oncol 2015;11:253-61.

159.Zinner R, Visseren-Grul C, Spigel DR, et al. Pemetrexed clinical studies in performance status 2 patients with non-small cell lung cancer (Review). Int J Oncol 2016;48:13-27.

160. Nakashima K, Akamatsu H, Murakami H, et al. Carboplatin Plus Nab-paclitaxel in Performance Status 2 Patients with Advanced Non-small-cell Lung Cancer. Anticancer Res 2019;39:1463-8.

161.Zwitter M, Kovac V, Rajer M, et al. Two schedules of chemotherapy for patients with non-small cell lung cancer in poor performance status: a phase II randomized trial. Anticancer Drugs 2010;21:662-8.

162. Bahij R, Jeppesen SS, Olsen KE, et al. Outcome of treatment in patients with small cell lung cancer in poor performance status. Acta Oncol 2019;58:1612-7.

163. Okuma Y, Hosomi Y, Nagamata M, et al. Clinical outcomes after first-line EGFR inhibitor treatment for patients with NSCLC, EGFR mutation, and poor performance status. Anticancer Res 2013;33:5057-64. 
164. Kashiwabara K, Fujii S, Tsumura S, et al. Overall survival of super-elderly ( 85 years or older) advanced non-small cell lung cancer patients with active epidermal growth factor receptor mutations receiving first-line gefitinib therapy: a single-institute retrospective study. J Cancer Res Clin Oncol 2021;147:287-93.

165. Nakashima K, Kimura M, Akamatsu H, et al. Osimertinib for patients with EGFR T790M mutation-positive nonsmall-cell lung cancer and a poor performance status. Jpn J Clin Oncol 2019;49:671-5.

166. Nakashima K, Ozawa Y, Daga H, et al. Osimertinib for patients with poor performance status and EGFR T790M mutation-positive advanced non-small cell lung cancer: a phase II clinical trial. Invest New Drugs 2020;38:1854-61.

167. Matsuo N, Sekine A, Kato T, et al. Promising Effect of Crizotinib on Anaplastic Lymphoma Kinase (ALK)Positive Non-Small Cell Lung Cancer in an Elderly Patient with a Poor Performance Status: A Case Report and Literature Review. Intern Med 2016;55:507-9.

168. Iwama E, Goto Y, Murakami H, et al. Alectinib for Patients with ALK Rearrangement-Positive Non-Small Cell Lung Cancer and a Poor Performance Status (Lung Oncology Group in Kyushu 1401). J Thorac Oncol 2017;12:1161-6.

169. Iwama E, Goto Y, Murakami H, et al. Survival Analysis for Patients with ALK Rearrangement-Positive NonSmall Cell Lung Cancer and a Poor Performance Status Treated with Alectinib: Updated Results of Lung Oncology Group in Kyushu 1401. Oncologist 2020;25:306-e618.

170. Masuda N, Ohe Y, Gemma A, et al. Safety and effectiveness of alectinib in a real-world surveillance study in patients with ALK-positive non-small-cell lung cancer in Japan. Cancer Sci 2019;110:1401-7.

171.Spigel DR, Hainsworth JD, Joseph MJ, et al. Randomized phase 2 trial of pemetrexed, pemetrexed/bevacizumab, and pemetrexed/carboplatin/bevacizumab in patients with stage IIIB/IV non-small cell lung cancer and an Eastern Cooperative Oncology Group performance status of 2. Cancer 2018;124:1982-91.

172. Riggs H, Jalal SI, Baghdadi TA, et al. Erlotinib and bevacizumab in newly diagnosed performance status 2 or elderly patients with nonsquamous non-small-cell lung cancer, a phase II study of the Hoosier Oncology Group: LUN04-77. Clin Lung Cancer 2013;14:224-9.

173.Lunacsek OE, Ravelo A, Coutinho AD, et al. First-Line Treatment with Bevacizumab and Platinum Doublet
Combination in Non-Squamous Non-Small Cell Lung Cancer: A Retrospective Cohort Study in US Oncology Community Practices. Drugs Real World Outcomes 2016;3:333-43.

174. Spigel DR, McCleod M, Jotte RM, et al. Safety, Efficacy, and Patient-Reported Health-Related Quality of Life and Symptom Burden with Nivolumab in Patients with Advanced Non-Small Cell Lung Cancer, Including Patients Aged 70 Years or Older or with Poor Performance Status (CheckMate 153). J Thorac Oncol 2019;14:1628-39.

175. Felip E, Ardizzoni A, Ciuleanu T, et al. CheckMate 171: A phase 2 trial of nivolumab in patients with previously treated advanced squamous non-small cell lung cancer, including ECOG PS 2 and elderly populations. Eur J Cancer 2020;127:160-72.

176. Middleton G, Brock K, Savage J, et al. Pembrolizumab in patients with non-small-cell lung cancer of performance status 2 (PePS2): a single arm, phase 2 trial. Lancet Respir Med 2020;8:895-904.

177. Katsura H, Suga Y, Araya T, et al. Efficacy and Safety of Nivolumab in Patients with Advanced Non-small-cell Lung Cancer and Poor Performance Status. J Cancer 2019;10:2139-44.

178. Alessi JV, Ricciuti B, Jiménez-Aguilar E, et al. Outcomes to first-line pembrolizumab in patients with PD-L1-high ( $\geq 50 \%$ ) non-small cell lung cancer and a poor performance status. J Immunother Cancer 2020;8:e001007.

179. Facchinetti F, Mazzaschi G, Barbieri F, et al. First-line pembrolizumab in advanced non-small cell lung cancer patients with poor performance status. Eur J Cancer 2020;130:155-67.

180. Fiorelli A, Perrotta F, Mollica M, et al. Endoscopic central airway recanalization to enable first line pembrolizumab treatment in a PD-L1 strongly positive non-small cell lung cancer: a case report. J Cardiothorac Surg 2019;14:50.

181. Wang Y, Zhang Q, Miao L, et al. Nivolumab in combination with anlotinib achieved remarkable efficacy in a patient with driver-negative lung squamous cell carcinoma and PS of 4. Ann Palliat Med 2020;9:4384-8.

182. Nie NF, Liu ZL, Feng MX, et al. Lazarus type response to immunotherapy in three patients with poor performance status and locally advanced NSCLC: a case series and literature review. Ann Palliat Med 2021;10:210-9.

183. Reck M, Socinski MA, Cappuzzo F, et al. Primary PFS and safety analyses of a randomized phase III study of 
carboplatin + paclitaxel +/- bevacizumab, with or without atezolizumab in $1 \mathrm{~L}$ non-squamous metastatic NSCLC (IMPOWER150). Ann Oncol 2017;28:xi31.

184. Levy BP, Signorovitch JE, Yang H, et al. Effectiveness of first-line treatments in metastatic squamous non-small-cell lung cancer. Curr Oncol 2019;26:e300-8.

185.Mccune S, Ton T, Whipple S, et al. EP1.12-06 New Treatment Option for ES-SCLC: Patient Characteristics and Use of an Atezolizumab Regimen in the Real-World Setting. J Thorac Oncol 2019;14:S1018-9.

186. Liu SV, Camidge DR, Gettinger SN, et al. Long-term survival follow-up of atezolizumab in combination with platinum-based doublet chemotherapy in patients with advanced non-small-cell lung cancer. Eur J Cancer 2018;101:114-22.

187. Pastores SM, Voigt LP. Acute respiratory failure in the patient with cancer: diagnostic and management strategies. Crit Care Clin 2010;26:21-40.

188. Rotman JA, Plodkowski AJ, Hayes SA, et al. Postoperative complications after thoracic surgery for lung cancer. Clin Imaging 2015;39:735-49.

189. Nava S, Ferrer M, Esquinas A, et al. Palliative use of non-invasive ventilation in end-of-life patients with solid tumours: a randomised feasibility trial. Lancet Oncol 2013;14:219-27.

190. Girou E, Schortgen F, Delclaux C, et al. Association of noninvasive ventilation with nosocomial infections and survival in critically ill patients. JAMA 2000;284:2361-7.

191.Segura A, Pardo J, Jara C, et al. An epidemiological evaluation of the prevalence of malnutrition in Spanish patients with locally advanced or metastatic cancer. Clin Nutr 2005;24:801-14.

192.Hébuterne X, Lemarié E, Michallet M, et al. Prevalence of malnutrition and current use of nutrition support in patients with cancer. JPEN J Parenter Enteral Nutr 2014;38:196-204.

193. Mariani L, Lo Vullo S, Bozzetti F, et al. Weight loss in cancer patients: a plea for a better awareness of the issue. Support Care Cancer 2012;20:301-9.

194.Na BG, Han SS, Cho YA, et al. Nutritional Status of Patients with Cancer: A Prospective Cohort Study of 1,588 Hospitalized Patients. Nutr Cancer 2018;70:1228-36.

195. Kiss N. Nutrition support and dietary interventions for patients with lung cancer: current insights. Lung Cancer (Auckl) 2016;7:1-9.

196.Arends J, Bachmann P, Baracos V, et al. ESPEN guidelines on nutrition in cancer patients. Clin Nutr
2017;36:11-48.

197. Guinan JL, McGuckin M, Nowell PC. Management of health-care--associated infections in the oncology patient. Oncology (Williston Park) 2003;17:415-20; discussion 423-6.

198. Wong JL, Evans SE. Bacterial Pneumonia in Patients with Cancer: Novel Risk Factors and Management. Clin Chest Med 2017;38:263-77.

199. Evans SE, Ost DE. Pneumonia in the neutropenic cancer patient. Curr Opin Pulm Med 2015;21:260-71.

200.Lyman GH, Khorana AA, Falanga A, et al. American Society of Clinical Oncology guideline: recommendations for venous thromboembolism prophylaxis and treatment in patients with cancer. J Clin Oncol 2007;25:5490-505.

201. Khorana AA, Noble S, Lee AYY, et al. Role of direct oral anticoagulants in the treatment of cancer-associated venous thromboembolism: guidance from the SSC of the ISTH. J Thromb Haemost 2018;16:1891-4.

202. Watson HG, Keeling DM, Laffan M, et al. Guideline on aspects of cancer-related venous thrombosis. $\mathrm{Br} \mathrm{J}$ Haematol 2015;170:640-8.

203. Key NS, Khorana AA, Kuderer NM, et al. Venous Thromboembolism Prophylaxis and Treatment in Patients With Cancer: ASCO Clinical Practice Guideline Update. J Clin Oncol 2020;38:496-520.

204.Lai Y, Su J, Yang M, et al. Impact and Effect of Preoperative Short-term Pulmonary Rehabilitation Training on Lung Cancer Patients with Mild to Moderate Chronic Obstructive Pulmonary Disease: A Randomized Trial. Zhongguo Fei Ai Za Zhi 2016;19:746-53.

205. Benzo R, Wigle D, Novotny P, et al. Preoperative pulmonary rehabilitation before lung cancer resection: results from two randomized studies. Lung Cancer 2011;74:441-5.

206. Sebio Garcia R, Yáñez Brage MI, Giménez Moolhuyzen E, et al. Functional and postoperative outcomes after preoperative exercise training in patients with lung cancer: a systematic review and meta-analysis. Interact Cardiovasc Thorac Surg 2016;23:486-97.

207.Sommer MS, Staerkind MEB, Christensen J, et al. Effect of postsurgical rehabilitation programmes in patients operated for lung cancer: A systematic review and metaanalysis. J Rehabil Med 2018;50:236-45.

208. Chen HM, Tsai CM, Wu YC, et al. Randomised controlled trial on the effectiveness of home-based walking exercise on anxiety, depression and cancerrelated symptoms in patients with lung cancer. $\mathrm{Br} \mathrm{J}$ 
Cancer 2015;112:438-45.

209. Quist M, Adamsen L, Rørth M, et al. The Impact of a Multidimensional Exercise Intervention on Physical and Functional Capacity, Anxiety, and Depression in
Patients with Advanced-Stage Lung Cancer Undergoing Chemotherapy. Integr Cancer Ther 2015;14:341-9.

210. Wang H, Liu X, Rice SJ, et al. Pulmonary Rehabilitation in Lung Cancer. PM R 2016;8:990-6.

Cite this article as: Zhou C, Li S, Liu J, Chu Q, Miao L, Cai L, Cai X, Chen Y, Cui F, Dong Y, Dong W, Fang W, He Y, Li W, Li M, Liang W, Lin G, Lin J, Lin X, Liu H, Liu M, Mu X, Hu Y, Hu J, Jin Y, Li Z, Qin Y, Ren S, Sun G, Shen Y, Su C, Tang K, Wu L, Wang M, Wang H, Wang K, Wang Y, Wang P, Wang H, Wang Q, Wang Z, Xie X, Xie Z, Xu X, Xu F, Yang M, Yang B, Yi X, Ye X, Ye F, Yu Z, Yue D, Zhang B, Zhang J, Zhang J, Zhang X, Zhang W, Zhao W, Zhu B, Zhu Z, Zhong W, Bai C, Chen L, Han B, Hu C, Lu S, Li W, Song Y, Wang J, Zhou C, Zhou J, Zhou Y, Saito Y, Ichiki Y, Igai H, Watanabe S, Bravaccini S, Fiorelli A, Petrella F, Nakada T, Solli P, Tsoukalas N, Kataoka Y, Goto T, Berardi R, He J, Zhong N. International consensus on severe lung cancer-the first edition. Transl Lung Cancer Res 2021;10(6):2633-2666. doi: 10.21037/tlcr-21-467 